\title{
Ad Honorem Yves Meyer
}

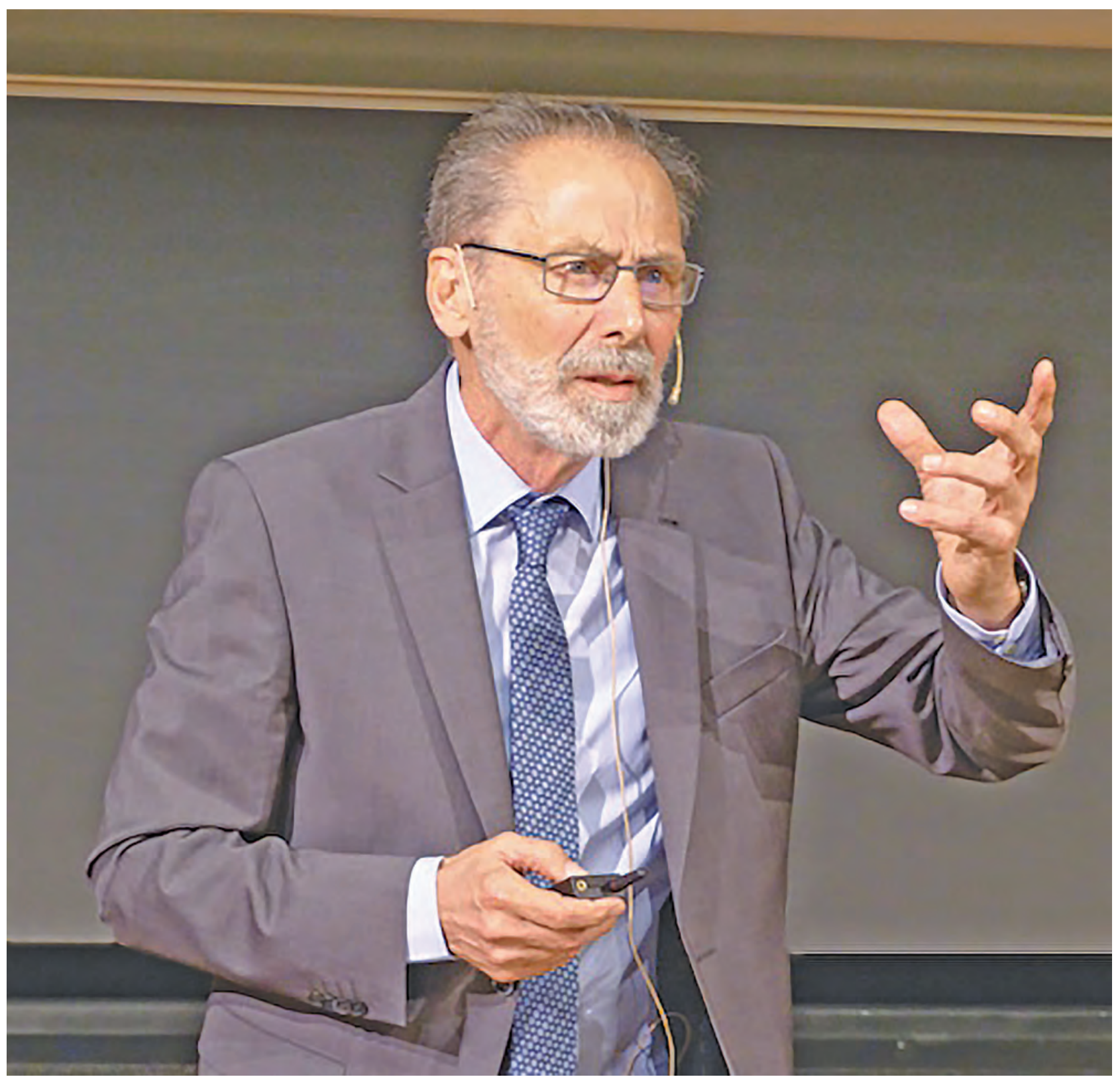

For permission to reprint this article, please contact:

reprint-permission@ams.org.

DOI: http://dx.doi.org/10.1090/noti1756 


\section{Ronald Coifman, Guest Editor}

\section{Introduction}

Yves Meyer was awarded the 2017 Abel Prize. His work has impacted mathematics in a broad and profound way. Perhaps even more importantly, he has led a broad, multifaceted, worldwide network of research collaborations of mathematics with music, chemistry, physics, and signal processing. He has made seminal contributions in a number of fields, from number theory to applied mathematics.

Meyer started his career on the interface between Fourier analysis and number theory. Early in his career he introduced the theory of model sets [1], which have become an important tool in the mathematical study of aperiodic order two years before the discovery of Penrose pavings by Roger Penrose and ten years before the discovery of quasi-crystals by Dan Shechtman.

Around 1975 he initiated the field of nonlinear Fourier analysis as a tool for organizing and analyzing nonlinear functional transformations of mathematics. He developed all the fundamental tools and concepts necessary to understand the nonlinear dependence of solutions of boundary value problems on the shape of the boundary. In particular, together with Coifman and McIntosh [4], he solved in 1982 the last outstanding problem of classical harmonic analysis by proving the continuity of the Cauchy integral operator on Lipschitz curves. It had been the key obstacle to the solvability of boundary value problems for Lipshitz domains (e.g. domains with corners). His methodologies prepared the way for Bony's para-differential calculus, Wu's proof of the existence of water waves in three dimensions, and the proof of Kato's conjecture, essentially changing the landscape of analysis.

Around 1984 Meyer [6]-[8] discovered the relation between the analytic tools used in harmonic analysis and various signal processing algorithms used in seismic exploration. In his Abel Prize interview [DS], Meyers said, "Morlet, Grossmann, and Daubechies were in a sense ahead of me in their work on wavelets. So I was the 'Quatrieme Mousquetaire.' They were Les Trois Mousquetaires.” He recognized their work as a rediscovery of Calderón's formulas in harmonic analysis, thereby bridging fifty years of multiscale harmonic analysis with "wavelets." This discovery led later to the construction of the Meyer wavelet basis, an orthonormal basis of functions localized in space and frequency. His work inspired Daubechies to discover the compactly supported orthonormal wavelet bases, which profoundly affected the field of engineering, leading in subsequent work to nonlinear adapted Fourier analysis and signal processing [3]-[5].

The technological impact has been remarkable. For example, the current JPEG 2000 standard for image compression has evolved from the wavelet tools invented by Meyer. The field of wavelet analysis has thousands

Ronald Coifman is professor of mathematics at Yale University. His email address is coifman@math. yale. edu. of papers in areas of application ranging from signal processing to medical diagnostics. Modern engineering depends on his methods. Numerical analysis uses his tools for efficient numerical computation of linear and nonlinear maps.

More recently Meyer has introduced new tools for analysis of the Navier-Stokes equations for fluid flow, discovering remarkable profound mechanisms relating oscillation to stability and blowups.

Some of Meyer's close collaborators have kindly provided some descriptions of his work, with a goal of covering a broad panorama of analysis. Stéphane Mallat, who formalized with Meyer the orthogonal multiresolution framework, describes Meyer's celebrated work on wavelets. His student Stéphane Jaffard (1989), who wrote the account [J] of his Abel Prize for the Société Mathématique de France Gazette des Mathématiciens, focuses on time-frequency analysis. Alexander Olevskii describes Meyer's Sets, which modeled quasi-crystals before they were discovered. My own contribution describes Meyer's work on nonlinear Fourier analysis. His student Albert Cohen (1990) describes Meyer's impact on sparse analysis.

Meyer's work is characterized by an extraordinary depth, solving longstanding problems, and starting new fields of mathematics and applications. These seminal, broad contributions have had a profound impact on different areas of science and establish him as a major figure in mathematics.

\section{References}

[DS] BJøRN IAN DUNDAS and CHRISTIAN SKAU, Interview with Abel Laureate Yves Meyer, Newsletter Eur. Math. Soc., Sept. 2017, 14-22, www.ems-ph.org /journa1s/news7etter/pdf/2017-09-105.pdf\#page=16, pp. 14-22; reprinted in the May 2018 Notices https: //www.ams.org/journa1s/notices/201805/rnoti-p520 .pdf. MR3726777

[J] S. JAFFARD, Yves Meyer, Prix Abel 2017 (French) Gaz. Math. No. 153 (2017), 20-26. smf4.emath.fr /Pub1ications/Gazette/2017/153/. MR3701591

\section{A Few Representative Works by Meyer}

[1] Algebraic Numbers and Harmonic Analysis, North Holland, New York (1972). MR0485769

[2] Wavelets and Operators, Vol. 1 and 2, revised version of 3, Cambridge Univ. Press (1992, 1997). MR1719426

[3] Wavelets, paraproducts and Navier-Stokes equations, in Current Developments in Mathematics 1996, MIT Press (1997).

[4] (with R. R. Coifman and A. McIntosh) L'intégrale de Cauchy sur les courbes Lipschitziennes, Annals of Math. 116 (1982) 361-387. MR672839

[5] (with Marco Cannone) Littlewood-Paley decompositions and Navier-Stokes equations, J. Methods Appl. Anal. (1995) 307319. MR1362019

[6] (with A. Grossmann, and I. Daubechies) Painless nonorthogonal expansions. Journal of Mathematical Physics 27, 1271 (1986). MR836025

[7] Wavelets and Applications, Proc. Int. Congr. Math. Kyoto (1990) 1619-1626. Math. Soc. Japan. 
[8] (with M. Farge, E. Goirand, F. Pascal, and V. Wickerhauser) Improved predictability of two-dimensional turbulent flows using wavelet packet compression, Fluid Dynamics Research (1992) 229-250.

\section{Photo Credit}

Opening photo courtesy of Stéphane Jaffard.

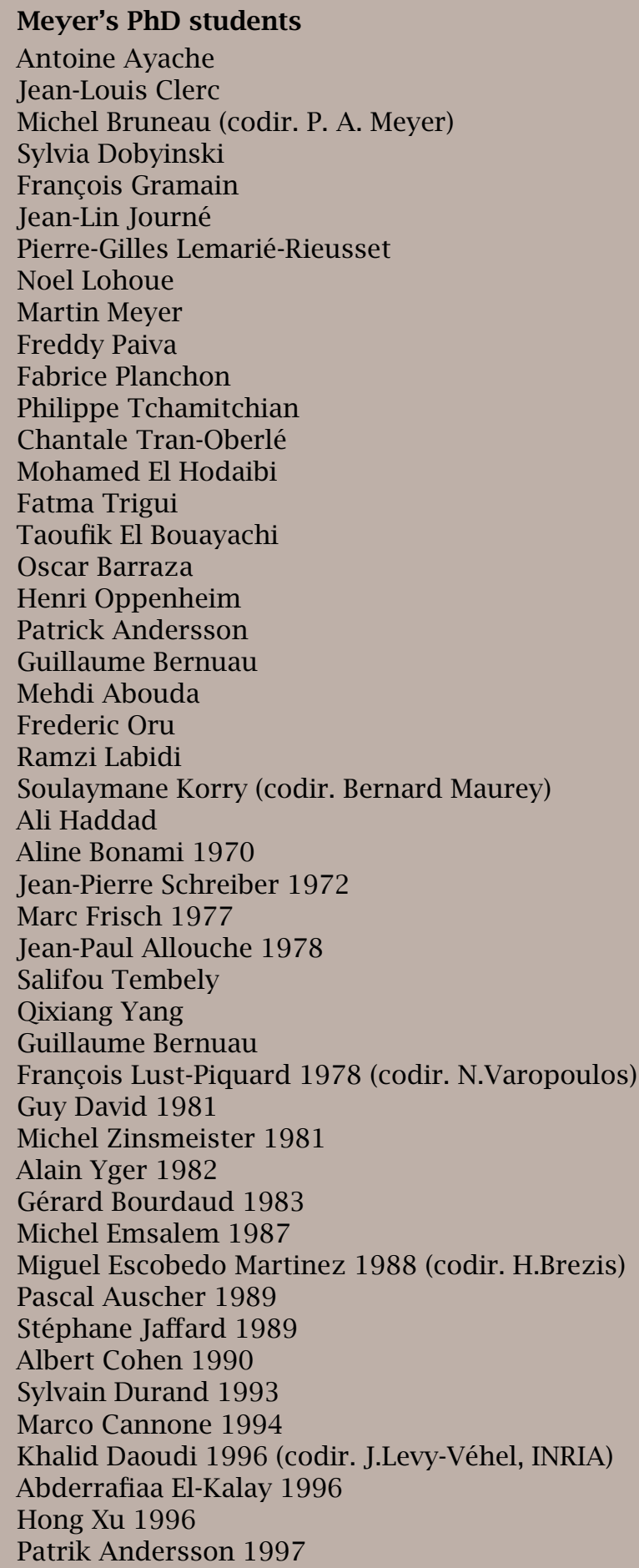

Lorenzo Brandolese 2001

Diego Chamorro 2006

Jérôme Gilles 2006

Xiaolong Li 2006

\section{Ronald Coifman}

Yves Meyer's Work on Nonlinear Fourier Analysis: Beyond Calderón-Zygmund

It is a privilege to be able to relate ideas, explorations, and visions that Yves, his collaborators, and his students developed over the last forty years, for some of which I was an active participant and observer.

We have had a lot of fun and excitement in this adventure, exploring and discovering beauty and structure.

I will focus my narrative on the simplest illustrations and examples of Yves Meyer's foundational contributions to nonlinear harmonic analysis, and try to illuminate and motivate some key programmatic issues that continue and build beyond the Calderón-Zygmund vision and program. It was Zygmund's view that harmonic analysis provides the infrastructure linking all areas of analysis, from complex analysis to partial differential equations to probability and geometry.

In particular he pushed forward the idea that the remarkable tools of complex analysis, such as contour integration, conformal mappings, and factorization, used to provide miraculous proofs in real analysis should be deciphered and converted to real variable tools. Together with Calderón, he bucked the trend for abstraction, prevalent at the time, and formed a school pushing forward this interplay between real and complex analysis. A principal bridge was provided by real variable methods, multiscale analysis, Littlewood Paley theory, and related Calderón representation formulas, later rediscovered by Morlet and others. They will be discussed here in relation to wavelets.

\section{Bilinear Convolutions, the Calderón Commutator, Complex Analysis, and Paraproducts}

In order to understand some of the basic ideas and methods introduced by Meyer and to illustrate the scope of the program, we start with the basic example of "paracalculus" introduced by Calderón as a bilinear operator needed to extend smooth pseudo-differential calculus to rough environments. He managed by an analytical tour de force using complex function theory to prove that the Calderón commutator defined below is a bounded operator on $L^{2}$.

Given a Lipschitz function $A$ on the real line (so $A^{\prime}:=a \in L^{\infty}(\mathbb{R})$ ) one formally defines the linear operator $C_{1}(f)$ by the formula

$$
\begin{aligned}
C_{1}(f) & =\int_{\mathbb{R}} \frac{A(x)-A(y)}{(x-y)^{2}} f(y) d y \\
& =(A|d / d x|-|d / d x| A) f=[A,|d / d x|](f),
\end{aligned}
$$

where the meaning of the absolute value of the derivative operator is given as

$$
|d / d x| f(x)=1 / 2 \pi \int_{\mathbb{R}} \exp (i x \xi)|\xi| \hat{f}(\xi) d \xi
$$


and $\hat{f}$ is the Fourier transform of $f$.

This is the first commutator of Calderón. The simplest particular case is obtained when $A(x)=x$ and $C_{1}(f)$ becomes the classical Hilbert transform. He introduced an auxiliary related operator, linking complex function theory and Fourier analysis as we now describe.

Let $a$ and $f$ be two periodic functions on the circle of power series type, with $a$ bounded and $f$ in $L^{2}$. Let $h$ satisfy $h^{\prime}=a f^{\prime}$.

Calderón's theorem is equivalent to the statement that $h$ is in $L^{2}$. Written in terms of Fourier coefficients of $a, f$, and $h$ this equation becomes

$$
\hat{h}_{k}=\sum_{0<j \leq k}(j / k) \hat{a}_{k-j} \hat{f}_{j} .
$$

Observe that $h(\theta)=\int_{0<t<\theta} a(t) f^{\prime}(t) d t$ and that the derivative is in the sense of distributions as $f$ is only in $L^{2}$. Meyer came out with the following-remarkably simple-proof. Write

$$
j / k=s=1 / \pi \int_{\mathbb{R}} s^{i \gamma} \frac{d \gamma}{1+\gamma^{2}} .
$$

Subsituting in (3) we get

$$
\hat{h}_{k}=1 / \pi \int_{\mathbb{R}} \sum_{0<j \leq k}(j / k)^{i \gamma} \hat{a}_{k-j} \hat{f}_{j} \frac{d \gamma}{1+\gamma^{2}},
$$

leading to the representation of $h$ as

$$
h=\pi(a, f)=1 / \pi \int_{\mathbb{R}} M_{-\gamma}\left(a M_{\gamma} f\right) \frac{d \gamma}{1+\gamma^{2}},
$$

where $M_{\gamma} f(\theta)=\sum_{0<k} k^{i \gamma} \hat{f}_{k} e^{i k \theta}$ is a contraction on $L^{2}$, proving that in the simpler case when $a$ is a bounded function and $f$ is in $L^{2}$ that $h$ is square integrable. Together we obtained the full strength of Calderón's theorem using real variable methods on $M_{-\gamma}$ as a singular integral operator on $H^{1}$.

An important property hidden in the paraproduct is the weak continuity of this bilinear expression. Observe that the product of functions is not a bilinear operation that is weakly continuous in the arguments: consider $\sin (n x)$, which converges weakly to 0 while $\sin (n x) \cdot \sin (n x)$ converges weakly to $1 / 2$. On the other hand, since each Fourier coefficient of the product of two functions of analytic type is a combination of a finite number of coefficients, it is clear that the paraproduct is weakly continuous in the arguments. The analysis done by Meyer and collaborators introduced real variable versions of the commutators or bilinear operators described above. These are fundamental building blocks for higher-dimensional nonlinear analysis, starting with bilinear pairings.

The simplest real variable version of the Calderón paraproduct is given in terms of wavelets or Haar functions as

$$
h=\pi(a, f)=\sum_{I} m_{I}(a)<f, h_{I}>h_{I},
$$

where $h_{I}$ are the Haar basis functions supported on the dyadic interval $I$ and $m_{I}(a)$ is the mean value of a, on that interval [2].

\section{Applications to Analytic Dependence}

One class of problems in nonlinear Fourier analysis concerns the nonlinear analytic dependence of linear operators on functional arguments. As we will see, such problems are deeply connected to all aspects of harmonic analysis [CM,4]. We shall focus on the seminal example of the Riemann mapping functional on rectifiable curves in the complex plane.

The fundamental mathematical question is whether this dependence is analytic on some families of curves. A particular example blending all of this is the following: We wish to understand the flow lines of water above a riverbed as in Figure 1, and their dependence on the modification of the shape of the bed. Since the flow lines are the images of horizontal lines under the Riemann mapping from the upper half plane to the region above the curve, we need to understand the dependence of the Riemann mapping on the curve. The link between the geometric description of the curve and the analytic problem is provided by the Cauchy transform. This operator is at the center of complex analysis, and provides a vehicle to understand the infinite-dimensional Banach manifold of rectifiable curves satisfying the chord-arc condition.

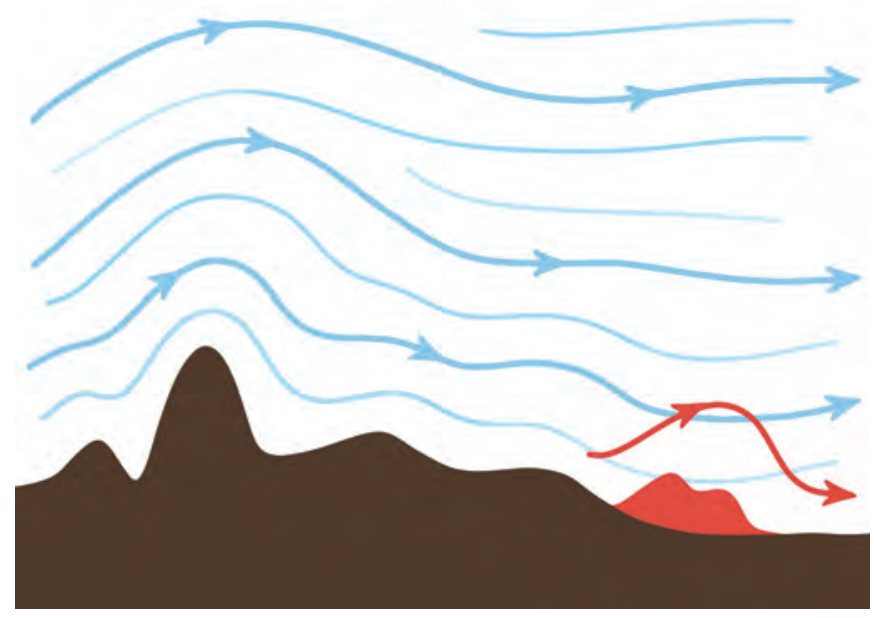

Figure 1. Flowlines above a riverbed. How are they going to be affected by the red bump?

So consider a Jordan curve in terms of its arc length parameterization, i.e.,

$$
z(s)=\int^{s} e^{i \alpha(t)} d t,
$$

and its corresponding Cauchy integral operator as:

$$
C(\alpha, f)(s)=p \cdot v \cdot(1 / 2 \pi i) \int_{\mathbb{R}} \frac{f(t) e^{i \alpha(t)} d t}{(z(s)-z(t))} .
$$

The natural space for which this operator-valued function of $\alpha$ is analytic is the space of functions of bounded mean oscillation (BMO). In fact the first derivative of $C(t \alpha, f)$ at $t=0$ is a bounded operator on $L^{2}$ if and only if $\alpha$ is in BMO. The condition of $\alpha(s)$ having a small norm in $\mathrm{BMO}$ is equivalent to the geometric chord-arc condition $|s-t|<(1+c)|z(s)-z(t)|$ for small $c$. 
We are now going to sketch the relationship of the question of analyticity to complex analysis, more surprisingly to operator functional calculus, and to the BMO manifold of chord-arc curves. This whole theory provides remarkable linkages between functional analysis, operator theory, singular integrals, and geometry.

Consider a monotone change of variable of the form $h(x)=\int^{x}(1+a(t)) d t$. We conjugate the operator $d / d x$ with the change of variable defined by $h(x)$ to obtain the operator $1 /(1+a) d / d x=U_{h} d / d x U_{h}^{-1}$, where $U_{h} f(x)=f\left(h^{-1}(x)\right)$. We now consider the operator $\operatorname{sgn}(d / d x)$ defined as

$$
\operatorname{sgn}(d / d x) f=1 / 2 \pi \int_{\mathbb{R}} \exp (i x \xi) \operatorname{sgn} \xi \hat{f}(\xi) d \xi .
$$

We let $h(x)=x+A(x)$ to conclude that

$$
\operatorname{sgn}\left((1 /(1+a) d / d x)=\int_{\mathbb{R}} \frac{f(t)(1+a(t)) d t}{x-t+(A(x)-A(t)} .\right.
$$

We see that the Cauchy integral for the curve above the graph of $A(x)$ is given by the same expression with $a$ replaced by $i a$.

More generally if we replace $a$ by $z a$, the analyticity in $a$ is equivalent to a bound in $c^{k}$ for the $k$-th Taylor coefficient in $z$, known as the $k$-th commutator. We also can show easily that the analytic dependence of the operator $\operatorname{sgn}((1 /(1+a) d / d x)$ on the coefficient $1 /(1+a)$ is equivalent to the boundedness of the Cauchy integral operator [1].

Returning to the Riemann mapping, we observe that the Cauchy transform is an oblique projection from $L^{2}$ onto the space of boundary restrictions (to the curve) of holomorphic functions on one side of curve. The corresponding orthogonal projection, called the Szego projection, can be easily expressed as a series involving the Cauchy transform, and also using the Riemann mapping itself, thereby leading to an analytic expression and a proof that the Riemann mapping depends analytically on the curve, provided that the Cauchy transform does. The distance between operators corresponding to two curves can be shown to be equivalent to the bounded mean oscillation norm of the difference between their arguments, thereby defining the most general geometry on the infinite-dimensional manifold of curves for which all of these objects are real analytic.

Several remarkable features appear here. The Cauchy transform on chord-arc curves is an operator-valued functional of the argument of the curve; it can be expanded in a convergent power series of operators on BMO. The norm on BMO is the operator norm defined by the first linear term. It defines the largest Banach space for which the nonlinear operator-valued transform is analytic.

Specifically for the Riemann map functional from the curve $z(s)=\int^{s} e^{i \alpha(t)} d t$ given in terms of the argument $\alpha(s)$ to the argument $\beta(s)$ of the change of variable $h(s)=\int^{s} e^{\beta(t)} d t$ defined by the Riemann map is an analytic functional, in the BMO topology of both $\alpha(s)$ and $\beta(s)$.

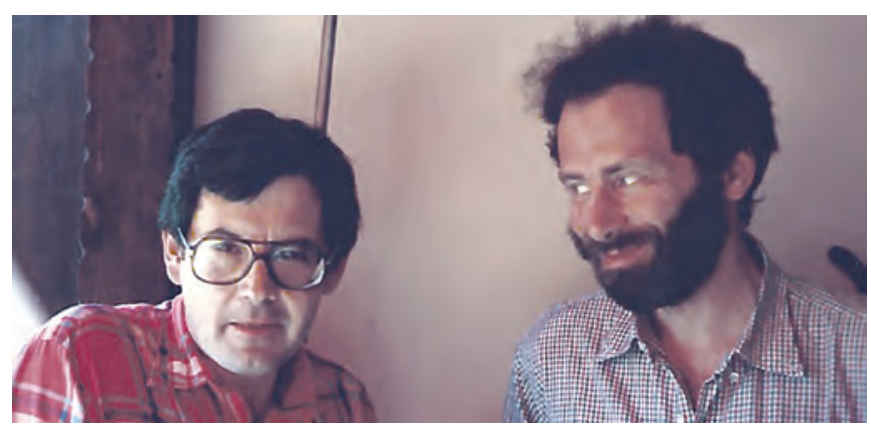

Figure 2. Coifman and Meyer having fun while working on the $k$ th commutators.

This theme of discovery of the natural functional space appears again in Meyer's work for the Navier-Stokes equations and other nonlinear PDE. The multilinear operators arising in the power series can be analyzed and decomposed directly, using Fourier or other transforms. This approach provides insight and could enable efficient numerical implementations. The Cauchy integral generalizes directly to higher dimensions, for example as a double layer potential operator, or more generally, as the restriction to a submanifold of a Calderón-Zygmund operator of the appropriate homogeneity. The long-standing problem of short-time existence of water waves in 2 or 3 dimensions was solved by Sijue Wu using these higherdimensional extensions. This example is related to a range of questions on the dependence and properties of functions of operators, or more generally on the dependence of spectral theory or resolvents on the coefficients.

The example of a variable-coefficient Laplace operator $\Delta$ in divergence form is related to the Kato conjecture, which states that the domain of $\Delta^{1 / 2}$ is the space of functions having a gradient in $L^{2}$. This conjecture in one variable is equivalent to the boundedness of the Cauchy integral, as proved by Coifman, McIntosh, and Meyer [1], and then extended to the general case close to the identity and finally resolved by Pascal Auscher, Steve Hofmann, Michael Lacey, Alan McIntosh, and Philippe Tchamitchian in 2002.

\section{A Few Representative Works by Meyer}

[1] R. R. Coifman, A. McIntosh, and Y. Meyer, L'integral de Cauchy définit un opérateur borné sur le courbes Lipschitziennes, Ann of Math., vol. 116, 361-387, 1982. MR672839

[2] R. R. COIFMAN and Y. MEYER, On commutators of singular integrals and bilinear singular integrals, Trans. Amer. Math. Soc., 315-331, 1975. MR0380244

[3] R. R. COIFMAN and Y. MEYER, Nonlinear harmonic analysis and analytic dependence. Proc. Symp. Pure Math 43, American Mathematical Society, Providence RI, 1985. MR812284

[4] R. R. COIFMAN and Y. MEYER, Wavelets: Calderón-Zygmund and multilinear operators, Cambridge Studies in Advanced Mathematics, 1997. MR1456993

[5] Y. MEYER, Wavelets and Operators, vol. 1, Cambridge University Press, 1997. MR1228209 
Image Credits

All section images courtesy of Ronald Coifman.

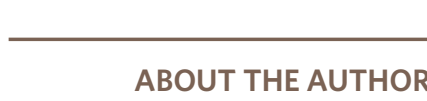

Ronald (Raphy) Coifman has been a close friend and collaborator of Yves Meyer for the last forty years, jointly encouraging a global network of harmonic analysts.

\section{Stéphane Mallat}

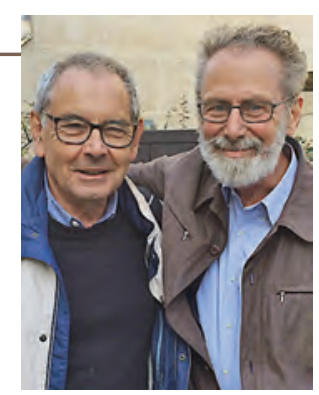

Ronald Coifman

\section{Orthogonal Wavelets: Bridging Beyond Mathe- matics}

Yves Meyer is a pure mathematician. This seemed obvious to us in the amphitheatre of 400 students at École Polytechnique in Paris, where Yves was enthusiastically describing the beauty of harmonic analysis as "a Mozart symphony." It was 1984, and Meyer already had the aura of an exceptional mathematician, with spectacular results in number theory and harmonic analysis. We thought that he was living in the limbo of abstractions, but we were wrong. I later realized that his intellectual home is transient, a nomad he sometimes calls himself, which is a root of his profound originality.

In 1986 it was under the umbrella of the Bourbaki seminar that Yves published his discovery of wavelet orthonormal bases, with $\mathbf{C}^{\infty}$ functions having a fast decay. This was not a 100-year-old conjecture, perhaps not looking spectacular for such a mathematical artist, but nonetheless opening a door to a world of science and mathematics. It led to more than a hundred thousand papers in many fields of science and mathematics, books, patents, industrial applications, and start-ups. He did not just open a door, but also penetrated the world of applications and led the way for a whole generation of mathematicians. Another spectacular outcome was to bridge pure and applied mathematics in France, the nation of Bourbaki.

But let me return to 1986. It was known that one could build an orthonormal basis of $\mathbf{L}^{2}(\mathbb{R})$ with functions that are dilations by $2^{j}$ and translations by $2^{j} n$ of a single real-valued function $\psi(t)$ for $(j, n) \in \mathbb{Z}^{2}$ :

$$
\left\{\psi_{j, n}(t)=2^{-j / 2} \psi\left(2^{-j}\left(t-2^{j} n\right)\right)\right\}_{(j, n) \in \mathbb{Z}^{2}} .
$$

As a result, any function $f \in \mathbf{L}^{2}(\mathbb{R})$ can be expanded as

$$
f=\sum_{(j, n) \in \mathbb{Z}^{2}}\left\langle f, \psi_{j, n}\right\rangle \psi_{j, n},
$$

Stéphane Mallat is professor of applied mathematics at the Collège de France. His email address is stephane . ma11 at@col1 ege -de-france. fr. where wavelet coefficients exhibit the local variations of $f$ at scale $2^{j}$ in the neighborhood of $t=2^{j} n$ :

$$
\left\langle f, \psi_{j, n}\right\rangle=\int f(t) \psi_{j, n}(t) d t .
$$

In 1909, Alfréd Haar constructed the first wavelet basis, generated by a piece-wise constant wavelet

$$
\psi=2^{-1 / 2}\left(1_{[0,1 / 2]}-1_{[1 / 2,1]}\right) .
$$

It is supported on $[0,1]$ and discontinuous.

It was also known that an orthonormal wavelet basis could be generated by the Shannon wavelet $\psi$ whose Fourier transform $\hat{\psi}(\omega)$ is compactly supported

$$
\widehat{\psi}=1_{[-2 \pi,-\pi]}+1_{[\pi, 2 \pi]} .
$$

The Shannon wavelet $\psi(t)$ is $\mathbf{C}^{\infty}$ but has a slow decay because its Fourier transform is discontinuous. The fact that it defines a wavelet orthonormal basis is closely related to the Shannon sampling theorem.

Yves knew about wavelets through his encounter with the physicist Alex Grossmann and the geophysicist Jean Morlet, who had introduced redundant families of regular wavelets with fast decay that spanned the space $\mathbf{L}^{2}(\mathbb{R})$ without being orthogonal. But is it possible to find a regular wavelet $\psi$ having a fast decay, which generates an orthonormal basis of $\mathbf{L}^{2}(\mathbb{R})$ ? In 1986 , this seemed highly unlikely otherwise it would certainly have been discovered before. The Balian-Low theorem proved that orthogonal bases composed of localized complex exponentials could not be constructed with regular functions having fast decay. To verify a similar property for wavelets, Yves tried to build an orthogonal basis with a regular and welllocalized wavelet, hoping to find the reason why it did not exist. To his surprise, he discovered wavelets $\psi$ that are $\mathbf{C}^{\infty}$ with a fast decay and that generate orthonormal bases. Meyer wavelets resemble Shannon wavelets, with a Fourier transform that has a compact support but that is $\mathrm{C}^{\infty}$. As a result $\psi(t)$ is also $\mathbf{C}^{\infty}$ with a fast decay: the best of both worlds, illustrated in Figure 1. Yves also extended this construction to define wavelet bases of functions of several variables, which led to important applications in image processing.

These wavelet constructions could have been anecdotal if Yves had not realized that these bases were unconditional bases of most classical functional spaces, from $\mathbf{L}^{\mathbf{p}}(\mathbb{R})$ for $p<1<\infty$ to Sobolev or Hölder spaces. Hence one can read the regularity of a function $f$ from the amplitude of its wavelet coefficients $\left|\left\langle f, \psi_{j, n}\right\rangle\right|$ and their decay as the scale $2^{j}$ goes to 0 . At fine scales, wavelet coefficients become very small in domains where $f$ is regular. If $f$ has few singularities, then at fine scales $2^{j}$ it has few large wavelet coefficients $\left\langle f, \psi_{j, n}\right\rangle$. One can thus approximate a piecewise regular function $f$ by keeping relatively few large coefficients $\left\langle f, \psi_{j, n}\right\rangle$ in its wavelet decomposition.

These properties opened the possibility to transport pure harmonic analysis to applications. It provided a mathematical framework to understand a wide range of scientific and industrial applications. But Yves did not 


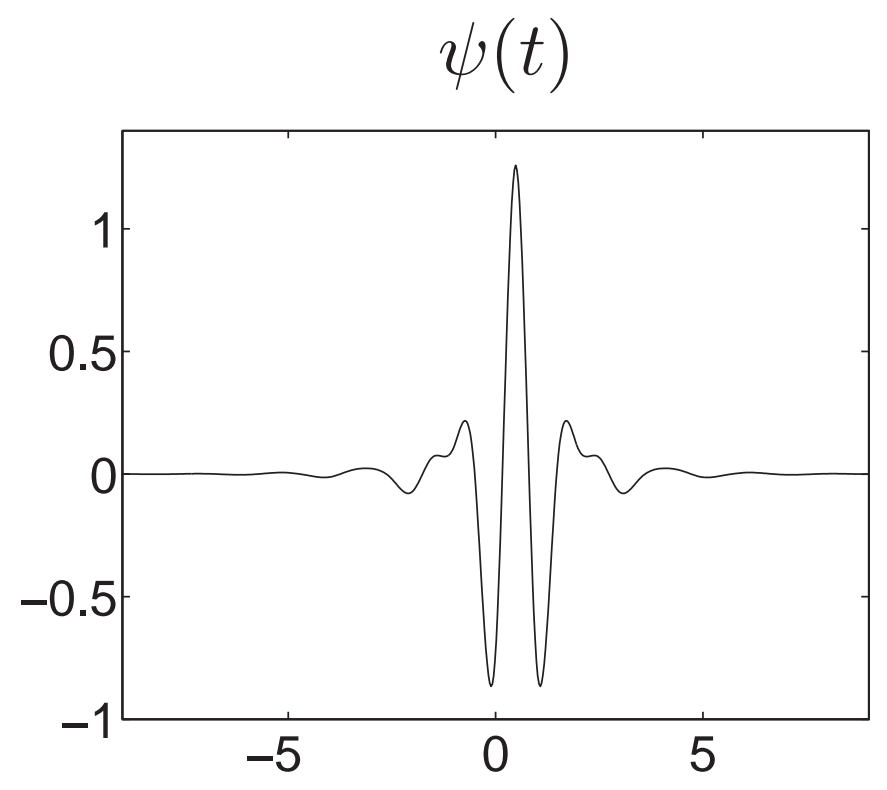

Figure 1. Graph of a Meyer wavelet. It is a $C^{\infty}$ function with a fast decay, whose translations and dilations define an orthonormal basis of $L^{2}(\mathbb{R})$.

stay in the cocoon of his native mathematics community, and crossed the door with enthusiasm. Similarly to Calderón and Zygmund in harmonic analysis, he became the concert master of a wide research program, providing a vision and connections between mathematics and multiple fields of science, and providing space for many researchers to contribute. This generosity drew a whole generation of young mathematicians along this path, without frontiers between pure and applied mathematics, science, and engineering applications. His student Pierre-Gilles Lemarié together with Guy Battle began by showing that other wavelet bases could be constructed with polynomial splines, having exponential decay.

a story where
mathematics and
other sciences
grew together

But this is not a story where the world was illuminated by mathematics, but where mathematics and other sciences grew together. Engineers had not been waiting arms crossed. Applications were too important. For telecommunications, you need to multiplex signals - to aggregate multiple signals into a single one for transmission and then be able to separate them. In 1977, two engineers, Esteban and Galand, had introduced a procedure to split $N$ coefficients of a sequence $x[n]$ for $0 \leq n<N$ into two sequences of $N / 2$ coefficients each, computed with a convolution of $x$ with two "filters" $h$ and $g$ while keeping one sample out of two:

$$
x_{1}[n]=x \star h[2 n] \text { and } x_{2}[n]=x \star g[2 n] .
$$

Finding conditions on $h$ and $g$ to recover $x$ from $x_{1}$ and $x_{2}$ became a major research issue in signal processing. Necessary and sufficient "conjugate mirror filter" conditions were established by Smith and Barnwell on the Fourier transforms $\hat{h}(\omega)$ and $\hat{g}(\omega)$ of the two filters:

$|\hat{h}(\omega)|^{2}+|\hat{h}(\omega+\pi)|^{2}=2$ and $\hat{g}(\omega)=e^{-i \omega} \hat{h}^{*}(\omega+\pi)$.

Although seemingly unrelated to wavelets, these filters then appeared to be at the core of wavelet orthonormal bases.

In computer vision, similar questions appeared in the 1980s, but for other reasons. To recognize objects in images, researchers proposed to detect edges, which appear at different scales. Reducing the resolution of images is important to eliminate fine details when they are not needed. In 1983, Peter Burt developed a pyramidal algorithm that iteratively averages the image with a filter $h$, while detecting multiscale edges with another filter $g$ derived from $h$.

In 1986, I had left École Polytechnique to do a $\mathrm{PhD}$ in image processing at the University of Pennsylvania. I was learning image pyramids and conjugate mirror filters when a friend gave me Meyer's paper on orthogonal wavelets. The connections became apparent. Multiresolution image pyramids could be formalized as projections in embedded subspaces of $\mathbf{L}^{2}(\mathbb{R})$, providing progressively finer approximations of functions as the scale decreases. Moreover, connection across scales appeared to be governed by the conjugate mirror filters $h$ and $g$ studied in signal processing, which also insure orthogonality properties. As a result, wavelets generating an orthonormal basis $\mathbf{L}^{2}(\mathbb{R})$ have a Fourier transform obtained by cascading the Fourier transform of these filters:

$$
\hat{\psi}(\omega)=2^{-1 / 2} \hat{g}\left(2^{-1} \omega\right) \prod_{p=2}^{\infty} \hat{h}\left(2^{-p} \omega\right) .
$$

This also implies that the wavelet coefficients $\left\langle f, \psi_{j, n}\right\rangle$ of a discretized function $f$ can be computed with a fast algorithm, by cascading convolutions and subsampling with $h$ and $g$, as in filter bank algorithms used by signal processing engineers. For appropriate filters, its requires fewer operations than the Fast Fourier Transform.

I sent my manuscript to Yves, who enthusiastically brought me to Chicago where he was working in the office of Zygmund. In three days, he solved all remaining mathematical problems. Yves had previously realized that there was an embedding-space structure underlying wavelets. We gave a sufficient condition to guarantee convergence in $\mathbf{L}^{2}(\mathbb{R})$ of the infinite filter product and obtain a wavelet that generates an orthonormal basis. This meant that new wavelet orthonormal bases could be constructed by defining filters that satisfy the conjugate mirror conditions discovered in signal processing. A necessary and sufficient condition was then found by Albert Cohen, a student of Yves, to relate wavelets to these filters.

At this point, many signal processing engineers looked at wavelets as a useless mathematical abstraction. What should they care about functions in $\mathbf{L}^{2}(\mathbb{R})$ when all 
computations are performed over finite sequences, with filters they had discovered before?

A first answer was given by the remarkable work of Ingrid Daubechies. Ingrid showed that more conditions had to be imposed on conjugate mirror filters to obtain regular wavelets of compact support. The Daubechies filters reduced computations, and the regularity of the resulting wavelets appeared to be important to avoid introducing visible artifacts when compressing images. In collaboration with Albert Cohen and Christian Feauveau, she designed the wavelet filters that have been adopted in the image compression standard JPEG-2000. This compression algorithm decomposes images in a wavelet basis and represents the non-zero coefficients with an efficient coding scheme. It can code images with 20 times fewer bits, with barely visible artifacts, because few wavelet coefficients of large amplitude need to be coded. The position of these large coefficients are located in near edges, as shown in Figure 2.

A second reason why mathematics became useful came from relations with Hölder regularity exponents, proved by Stéphane Jaffard, another student of Yves. He found necessary conditions and sufficient conditions to characterize the regularity of a function $f$ at any point $t$, from the decay of wavelet coefficients in a neighborhood of this point. It gave a mathematical framework to understand relations between wavelet coefficients and signal properties and to analyze very irregular functions such as multifractals.

A surprising connection appeared with statistics. David Donoho and Iain Johnstone realized that the unconditional basis properties proved by Meyer were exactly what was needed to suppress additive noise from signals having sparse wavelet coefficients. They showed that a simple thresholding, which set to zero the smallest wavelet coefficients of a noisy signal, is a nearly optimal non-linear estimator over large classes of signals. It opened a new field in non-linear statistics, which is still alive today.

Applications in numerical analysis came from the work of Gregory Beylkin, Raphy Coifman, and Vladimir Rocklin, who proved that many singular operators are represented by sparse matrices in a wavelet orthonormal basis. Matrix multiplications can then be computed with far fewer operations, for applications to calculations of solutions of partial differential equations, integral equations, and variational problems.

Wavelet orthonormal bases have found a multitude of applications, in chemistry, physics, many branches of information processing, and mathematics. They provided new tools in statistics and approximation theory, to specify multiscale properties of random processes or to develop fast numerical analysis algorithms. This is how the free nomadism of a curious and incredibly talented mathematician has had such high impact and created unexpected openings between many fields within and outside mathematics.

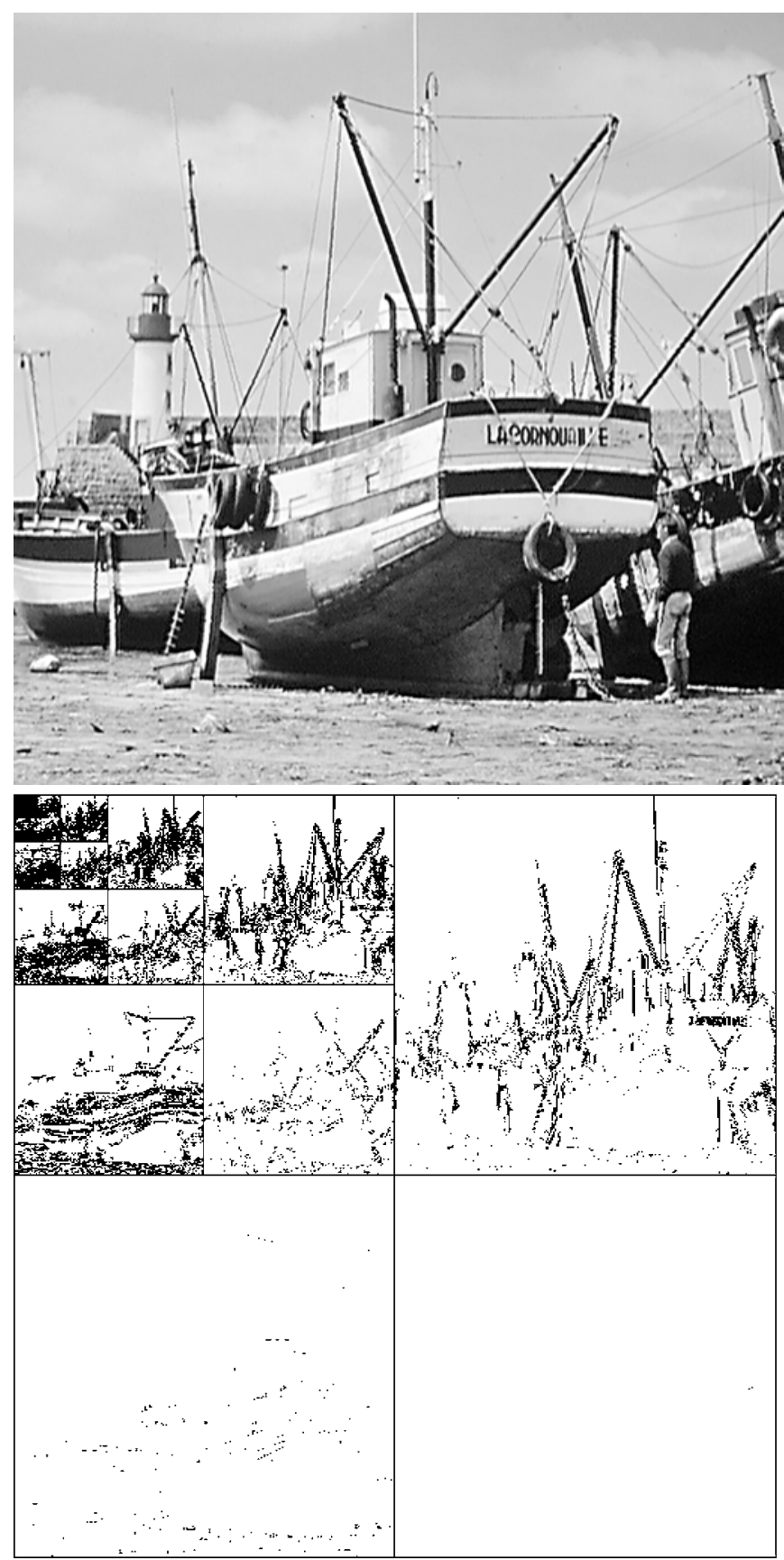

Figure 2. Top: original image $f$. It is an array of pixels that encode the image intensity. Bottom: each small image displays as dark points the wavelet coefficients that have a large amplitude, at a particular scale and orientation. Large coefficients are located where the image intensity has a sharp transition, near contours. Most coefficients are nearly zero (white), which is why they are compressed with much fewer bits than the original image pixels. 


\section{Image Credits}

All section images courtesy of Stéphane Mallat.

\section{ABOUT THE AUTHOR}

Stéphane Mallat works in mathematics applied to data analysis and machine learning, with applications to image and audio recognition, and learning models of physical systems.

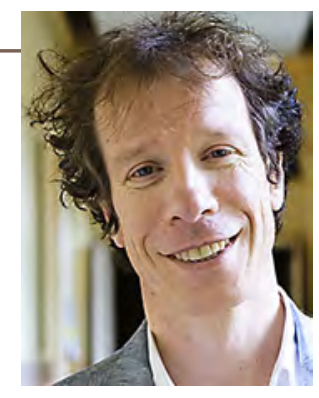

Stéphane Mallat

\section{Stéphane Jaffard}

\section{Time-Frequency Analysis, Chirps, and Local Regularity}

By the middle of the twentieth century the limitations of classical Fourier analysis for signal processing were patent. For example, musical recordings, which are a succession of notes of limited duration, clearly call for a localized Fourier analysis, as do chirps, a generic denomination which covers signals $f$ that locally look like a pure frequency, evolving slowly and smoothly with time. These defining conditions for chirps can be formalized as

$$
f(t)=\operatorname{Re}\left(a(t) e^{i \varphi(t)}\right),
$$

where the modulation factor $a$ and the instantaneous frequency $\varphi$ satisfy

$$
\left|\frac{a^{\prime}(t)}{a(t)}\right|<<\varphi^{\prime}(t) \text { and }\left|\varphi^{\prime \prime}(t)\right|<<\left(\varphi^{\prime}(t)\right)^{2} .
$$

The most important examples of chirps are supplied by gravitational waves (Figure 1): The very first one detected, in September 2015, was of the form $\left|t-t_{0}\right|^{-1 / 4} \cos (\omega \mid t-$ $\left.\left.t_{0}\right|^{5 / 8}+\eta\right)$.

Analysis of such data should involve time-frequency analysis. The signal is first localized by multiplying it with "windows." Gaussians are a natural choice because of their optimal localization in space and frequency. Then a Fourier analysis of the localized signal is performed. This idea was introduced in the 1940s by Nobel laureate D. Gabor and leads to the short-time Fourier transform of a function $f$, defined as

$$
G_{f}(x, \xi)=\int_{\mathbb{R}} f(t) \varphi(t-x) e^{-2 i \pi t \xi} d t,
$$

where $\varphi$ is the window. A continuous transform is computationally inefficient, and this raises the question of the existence of appropriate orthogonal decompositions, and of the corresponding fast transforms. Orthonormal bases cannot follow directly by sampling $G_{f}$ because of the Balian-Low theorem (1981), which states that if a

Stéphane Jaffard is professor of mathematics at Université Paris Est, France. His email address is jaffard@u-pec.fr.
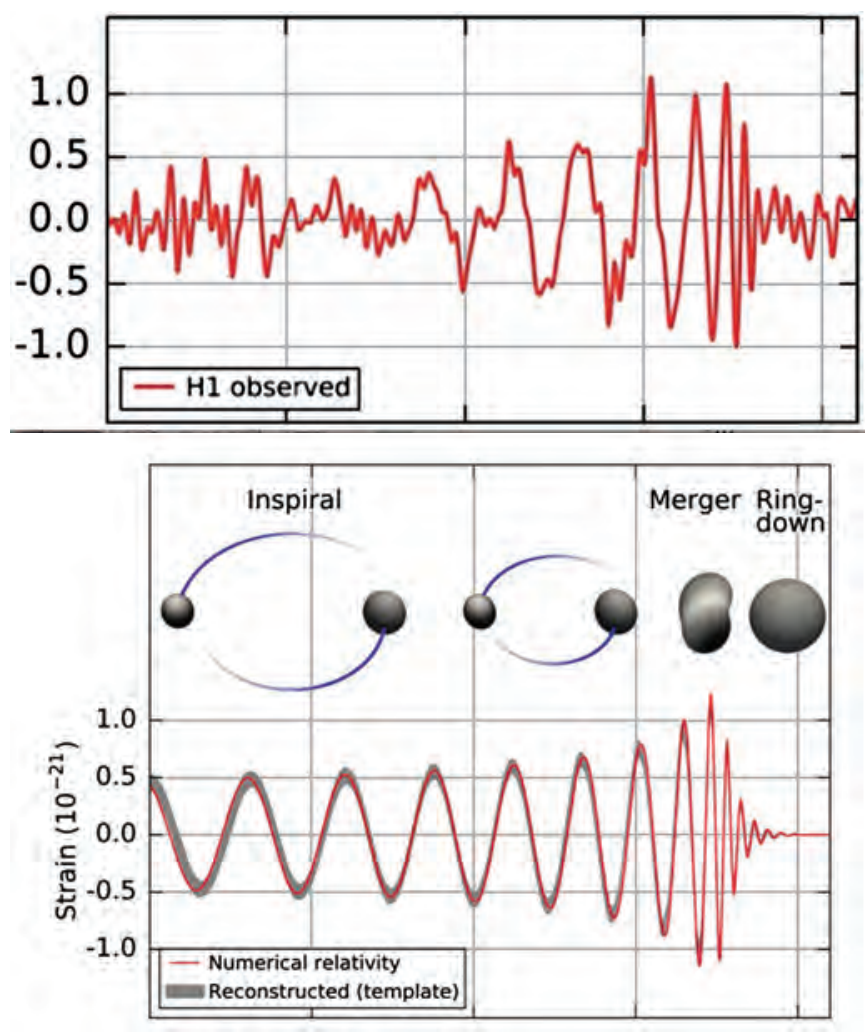

Figure 1. The gravitational wave as recorded by LIGO on September 14, 2015, after denoising; and the corresponding chirp as predicted by general relativity.

window $\varphi$ is both smooth and well localized then, for any choice of $a$ and $b$, a system of the form

$$
\varphi(t-a k) e^{i b n t} \quad k, n \in \mathbb{Z}
$$

is either incomplete or over-complete. Another Nobel laureate, K. Wilson, suggested a way to overcome this obstruction, by allowing for a double localization around opposite frequencies. Such bases, where the complex exponentials are simply replaced by sines and cosines, were constructed by Daubechies, Jaffard, and Journé and supply orthonormal bases of $L^{2}(\mathbb{R})$ of the form:

$$
\varphi_{n, k}(t)=\left\{\begin{array}{cc}
\varphi_{0, k}(t)=\varphi(t-k) \quad k \in \mathbb{Z}, \\
\sqrt{2} \varphi\left(t-\frac{k}{2}\right) \cos (2 \pi n t) \quad \text { if } k+n \in 2 \mathbb{Z}, \\
\sqrt{2} \varphi\left(t-\frac{k}{2}\right) \sin (2 \pi n t) & \text { if } k+n \in 2 \mathbb{Z}+1 .
\end{array}\right.
$$

S. Klimenko, who was the designer of Coherent Waveburst, the algorithm used in the signal processing part of the gravitational wave detection, chose this basis because it meets the following requirements:

- The window $\varphi$ can be Meyer's [LM] scaling function (Figure 2). This choice is motivated by the fact that a window with compact support in the Fourier domain allows elimination of noise components 


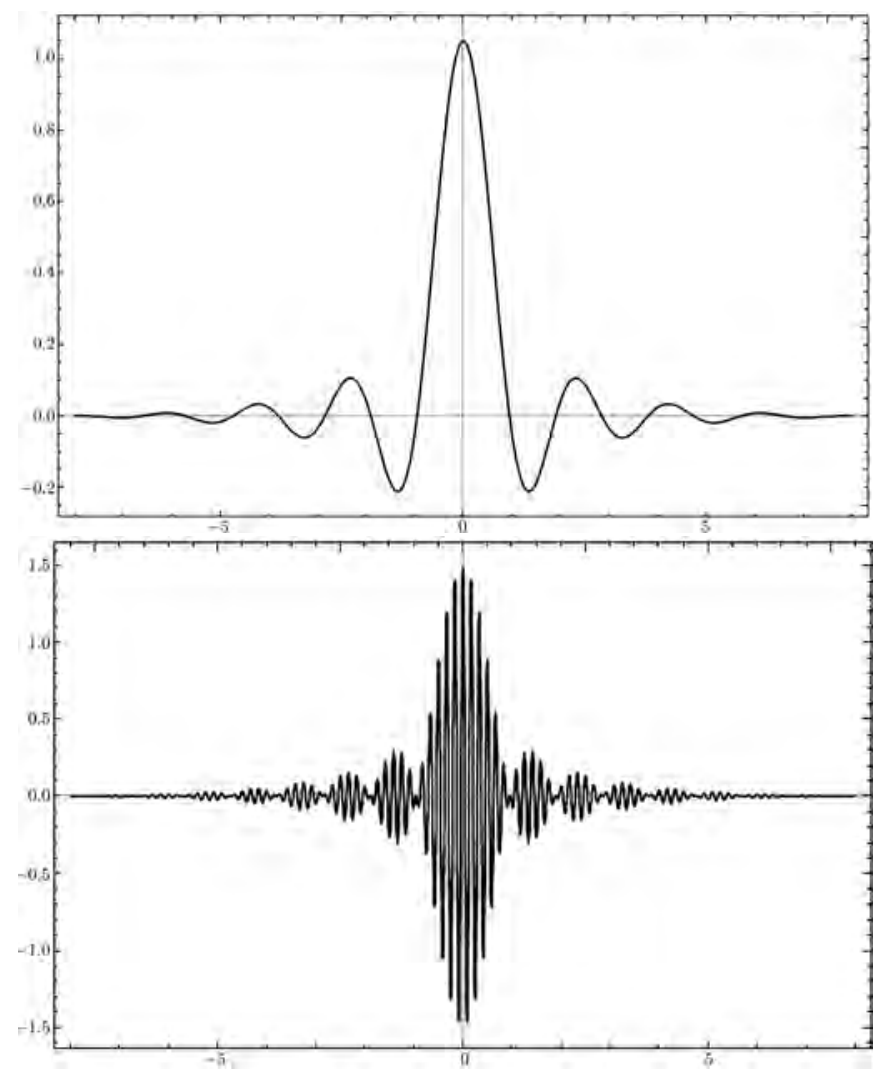

Figure 2. Meyer's scaling function and an element of the corresponding Wilson basis. Meyer's scaling function stands at the junction between two major extensions of Fourier analysis at the end of the twentieth century, time-scale and time frequency analysis. It is the building stone that allows generation of orthonormal bases in both settings.

that lie away from the main Fourier area of interest.

- Fast decomposition algorithms.

- Fast translation algorithms: one needs to compare the two signals recorded in the two LIGO detectors, which do not arrive exactly at the same time, and therefore need to be shifted.

- Gravitational waves are sparse in Wilson bases.

This procedure is now referred to as the WilsonDaubechies-Meyer transform.

Variants of Wilson bases were independently constructed by $H$. Malvar. They also are time-frequency orthonormal bases of $L^{2}(\mathbb{R})$ of the form

$$
\varphi_{n, k}(t)=\varphi(t-k) \cos \left[\pi\left(n+\frac{1}{2}\right)(t-k)\right] \quad k, n \in \mathbb{Z} .
$$

The corresponding decomposition, called MDCT (Modified Discrete Cosine Transform), is currently used in audio compression formats, e.g. MP3 or MPEG2 AAC. Malvar bases of adaptive lengths were introduced by R. Coifman and Y. Meyer [CM]: They are of the form

$$
\varphi_{n, k}(t)=\varphi_{k}(t) \cos \left[\frac{\pi}{l_{k}}\left(n+\frac{1}{2}\right)\left(t-a_{k}\right)\right],
$$

where the windows $\varphi_{n, k}$ start at $a_{k}$ and have arbitrary lengths $l_{k}$. These bases found a remarkable application in speech segmentation: E. Wesfreid and V. Wickerhauser devised an entropy minimization criterion that allows the lengths of the windows to adapt to the changes in the signal and thus performs automatic segmentation.

A different point of view can be developed for modeling chirps, where the instantaneous frequency $\varphi(t)$ in (4) diverges at a point $x_{0}$, leading to functions that have a singularity at $t_{0}$. This leads to pointwise singularities that are typically of the form

$$
C_{h, \beta}(t)=\left|t-t_{0}\right|^{h} \sin \left(\frac{1}{\left|t-t_{0}\right|^{\beta}}\right) .
$$

Based on the heuristic supplied by such toy-examples, Meyer [JM1] developed a general framework for such behaviors, where the sine function is replaced by a fairly arbitrary oscillating function, and showed that they are characterized by precise estimates on the wavelet coefficients. A remarkable application is supplied by the analysis of Riemann's function

$$
\mathcal{R}(t)=\sum_{n=1}^{\infty} \frac{\sin \left(\pi n^{2} t\right)}{n^{2}},
$$

which was proposed by Riemann as a candidate for a continuous nowhere differentiable function. It took a century to disprove Riemann's intuition and show that $\mathcal{R}$ is differentiable at rational points of the form $(2 p+$ 1) / $(2 q+1)$ (J. Gerver, 1970). Meyer considerably improved our comprehension of the behavior of Riemann's function in the neighbourhood of these points by exhibiting a complete chirp expansion about $t_{0}=1$ :

$\mathcal{R}(1+t) \sim-\frac{t}{2}+\sum_{k \geq 1}|t|^{k+1 / 2} g_{k}\left(\frac{1}{t}\right) \quad$ where $g_{k} \sim \mathcal{R}^{(-k)}$

(see Figure $3 ; g_{k}$ is essentially a primitive of order $k$ of the Riemann function itself!).

These explorations were the first stones that paved the way towards a classification of the pointwise singularities of everywhere irregular functions, now referred to as multifractal functions. Meyer [M1], [M2] introduced a new regularity exponent, the weak scaling exponent, which has the remarkable property of being covariant with respect to fractional primitives or derivatives. He revisited Wilson bases, constructed new bridges with wavelet decompositions, and built a whole variety of bases that can be tailored to particular chirp behaviors. With Jaffard [JM2] he uncovered an unexpected relationship between sparsity in a wavelet basis and pointwise regularity: They showed that generically, in the sense of Baire category, functions that have sparse wavelet expansions (the decreasing rearrangement of the sequence of their wavelet coefficients has fast decay) are multifractal, and they determined their multifractal spectrum (i.e. the Hausdorff dimensions of their pointwise Hölder singularities); Jaffard, Meyer, and Ryan [JMR] provide a user-friendly review on all these topics.

Meyer has been at the center of the effervescent and prolific multidisciplinary network that made the success of wavelets. He is a living proof that the limits between 

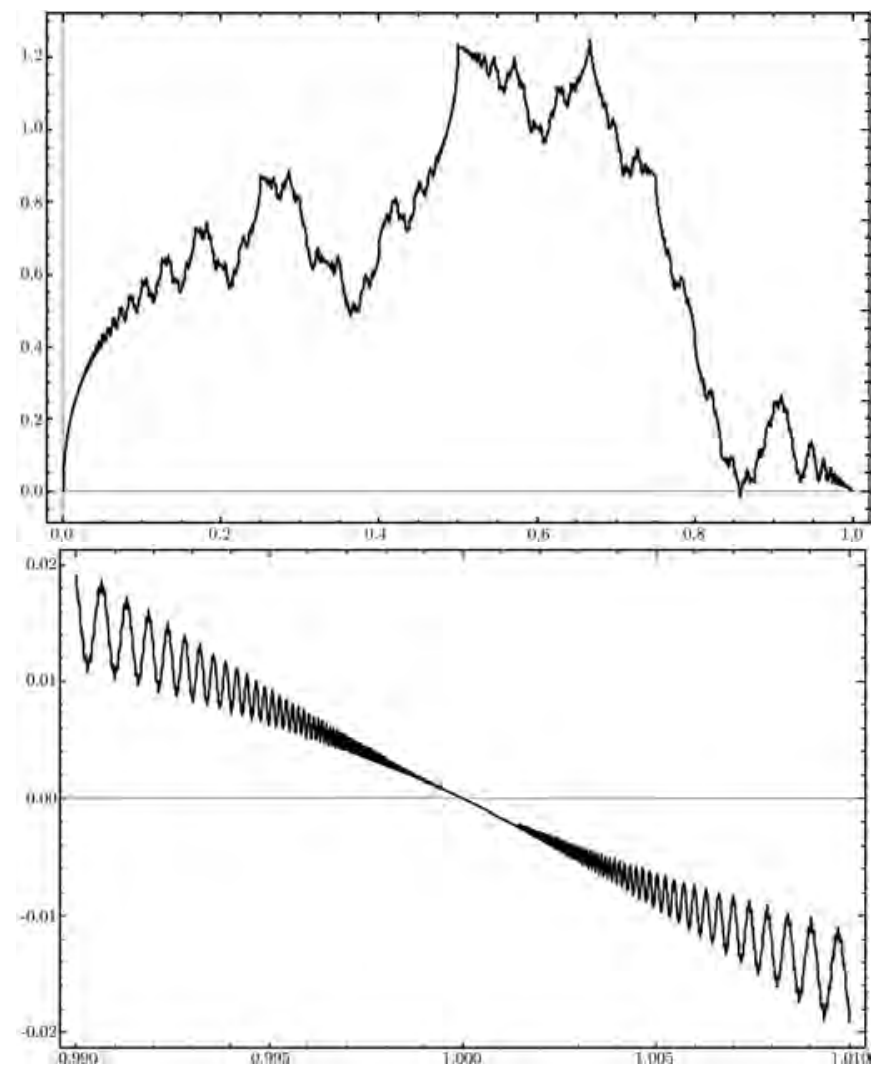

Figure 3. The Riemann function, and a zoom around the chirp at $t_{0}=1$. Meyer exhibited a complete chirp expansion near the rare differentiable points.

pure and applied science do not exist, and he repeatedly has shown that deep mathematical concepts can be the key to spectacular applications. He is famous for being a passionate lecturer. Extremely generous, he always pushed his many students and collaborators to the front; each of them can testify to the importance he laid to the transmission of science and the values of intellectual integrity, humanism, and tolerance. In a time driven by material values and short range-profit, Meyer stands as an example for younger generations of scientists.

\section{Cited Papers of Meyer}

[CM] R. COIFMAN and Y. MEYER, Remarques sur l'analyse de Fourier à fenêtre, C. R. Acad Sci. Paris, Sér I, Math. Vol. 312, pp. 259-261(1991). MR1089710

[JM1] S. JAFFARD and Y. MEYER, Wavelet methods for pointwise regularity and local oscillations of functions, Memoirs of the AMS, Vol. 123, no. 587 (1996). MR1342019

[JM2] S. JAFFARD and Y. MEYER, On the pointwise regularity of functions in critical Besov spaces, Vol. 175, N. 2, pp. 415-434 (2000). MR1780484

[JMR] S. JAFFARD, Y. MEYER and R. RYAN, Wavelets, tools for Science and Technology, SIAM (2001). MR1827998

[LM] P.-G. LEMARIÉ and Y. MEYER, Ondelettes en bases hilbertiennes, Revista Matematica Iberoamericana Vol. 2, pp. 1-18 (1986). MR864650

[M1] Y. MEYER, Wavelets, Vibrations and Scalings, CRM Monograph Series, Vol. 9, AMS (1998). MR1483896
[M2] Y. MEYER, Oscillating patterns in image processing and nonlinear evolution equations, University Lecture Series, Vol. 22, AMS (2000). MR1852741

\section{Image Credits}

Figure 1 dx. doi .org/10.7935/K5MW2F23.

Figures 2, 3, and author photo courtesy of Stéphane Jaffard.

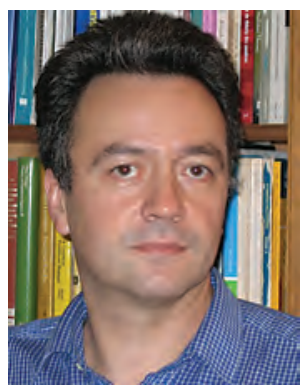

Stéphane Jaffard
ABOUT THE AUTHOR

Stéphane Jaffard did his PhD under the supervision of Yves Meyer at the very beginning of wavelet theory. His main subject of research is harmonic and multifractal analysis and their applications in signal and image processing.

\section{Alexander Olevskii}

\section{Meyer's Sets and Related Problems}

Many years ago Yves Meyer introduced remarkable concepts and constructions, which allowed him to solve some important problems in harmonic analysis. After many years these concepts and ideas remain important and are applicable to new problems.

Below I am going to focus on some aspects of this new development, in which Meyer is one of the main actors.

\section{Poisson Summation Formula}

We start with the classical Poisson formula:

$$
\sum_{\lambda \in \Lambda} \hat{f}(\lambda)=\frac{1}{|\Lambda|} \sum_{\lambda^{*} \in \Lambda^{*}} f\left(\lambda^{*}\right) .
$$

Here $\Lambda$ is a lattice in $\mathbb{R}^{n},|\Lambda|$ is the volume of its fundamental parallelepiped, $\Lambda^{*}$ is the dual lattice, $f$ is any function in the Schwartz class $S(\mathbb{R})$, and $\hat{f}$ its Fourier transform.

Equivalently, if $\mu$ is the sum of unit masses on a lattice (a Dirac comb), then its Fourier transform $\hat{\mu}$ (in sense of distributions) is again a Dirac comb. The spectrum of $\mu$ $(:=$ the support of $\hat{\mu})$ in this case is the dual lattice.

The Poisson formula has many applications. In particular, it plays a seminal role in the $X$-ray diffraction.

The following problem is important: do there exist some other discrete measures $\mu$ such that the distributional Fourier transform $\mu$ is also a discrete measure? Here one looks for measures $\mu$ that are not finite sums of Dirac combs, translated and modulated. This problem was studied in the 1950 s by J.-P. Kahane - S. Mandelbrot and by J.-P. Guinand.

Alexander Olevskii is professor of mathematics at Tel Aviv University. His email address is olevski i@post. tau.ac. i1. 


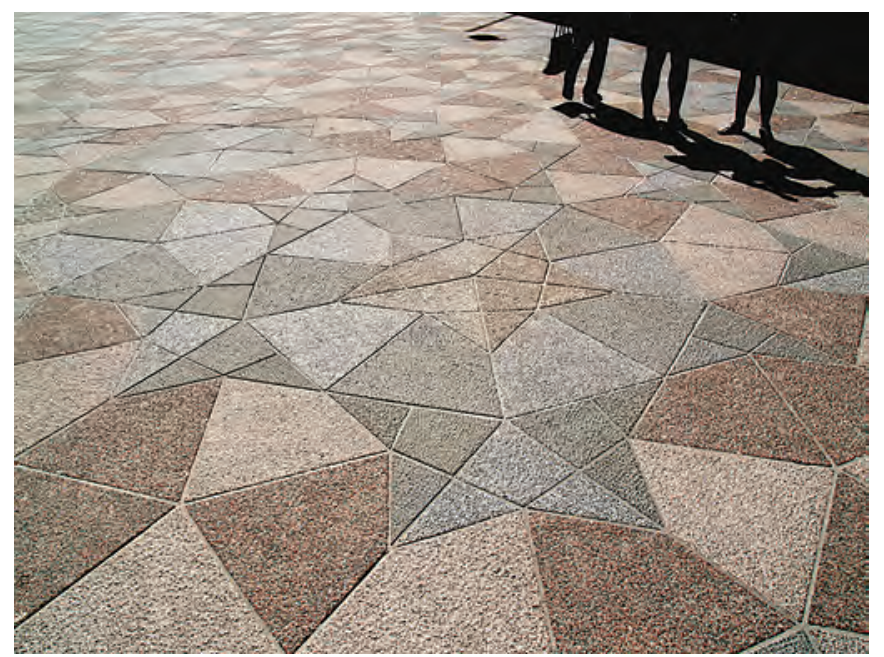

Figure 1. The Voronoi cells of a model set provide a nonperiodic tiling, such as this Penrose tiling in Keskuskatu Square, Helsinki.

\section{Meyer's Model Sets}

Yves Meyer [1] discovered a family of non-periodic discrete measures with point spectrum.

The construction is based on a concept of model sets, obtained from lattices by the cut-and-project procedure.

Here is the definition in the simplest case. In the plane $(x, y)$, take a lattice $\Gamma$ in the general position. Fix a set $Q$ (a "window") that is a finite union of intervals on the $y$-axis. Consider the set of lattice points that lie in $\mathbb{R} \times Q$, and project them onto the $x$-axis. This way one gets a Meyer model set $M$ in $\mathbb{R}$. A similar construction can be done in $\mathbb{R}^{n}$, starting with a lattice $\Gamma$ in $\mathbb{R}^{n} \times \mathbb{R}^{m}$.

Here are some properties of these model sets $M$ :

- Every $M$ is a nonperiodic uniformly discrete set. Uniform discreteness means that the pairwise distances between the different elements are separated from zero.

- The "Voronoi cells" corresponding to $M$ provide a tiling of $\mathbb{R}^{n}$ by non-overlapping translates of a finite family of polyhedra. A well-known example of such a tiling is due to Penrose (Figure 1). De Bruijn proved that the Penrose tiling can be obtained by the cut-and-project procedure from a 5-D lattice.

- Every model set $M$ supports a measure $\mu$ with point spectrum.

Here is a proof of the last property in the simplest case: let $M$ be a 1-D model obtained from a lattice $\Gamma$ and window $I$. Take a function $f \in S(\mathbb{R})$ supported on $I$. Set

$$
\mu:=\sum_{x, y \in \Gamma} f(y) \delta_{x} .
$$

Then $\mu$ is supported on $M$. Using the Poisson formula in $\mathbb{R}^{2}$ one can verify that

$$
\hat{\mu}=\sum_{(u, v) \in \Gamma^{*}} \hat{f}(v) \delta_{u} .
$$

So, $\hat{\mu}$ is a discrete measure with a dense support $S$.

\section{Model Sets and Reconstruction of Signals}

Here I discuss a new application of the model sets as sampling sets.

For a bounded set $S \subset \mathbb{R}$, denote by $P W_{S}$ (the PaleyWiener space with spectrum $S$ ) the space of functions (signals) $f \in L^{2}(\mathbb{R})$ whose Fourier transform is supported on $S$.

Given a uniformly discrete set $\Lambda$, one wishes to recover every $f \in P W_{S}$ from its values (samples) on $\Lambda$.

The following inequality would allow one to solve this problem in a stable way:

$$
\|f\|^{2} \leq C(S, \Lambda) \sum_{\lambda \in \Lambda}|f(\lambda)|^{2}, \quad \forall f \in P W_{S} .
$$

Given $S$, for which sets $\Lambda$ does this condition hold? When $S$ is an interval, A. Beurling proved that the validity of (2) is essentially equivalent to the condition that $\Lambda$ must be "dense" in a certain sense. H. Landau extended the necessity of the density condition to the case of disconnected spectra. However, in general no reasonable sufficient condition can be expressed in terms of some density of $\Lambda$. The geometric structure of $S$ plays a crucial role. Hence, a special construction of a "sampling set" $\Lambda$ is needed in the general case.

Is it possible to find $\Lambda$ so that (2) holds for every spectrum $S$ of given measure, independently of its structure and localization? This problem was put forward by A. Olevskii and A. Ulanovskii, who proved in 2006 that for compact spectra such "universal sampling sets" do exist. Such sets were constructed as certain perturbations of lattices. A new remarkable proof of this result was found in 2008 by B. Matey and Y. Meyer, who showed that appropriate model sets satisfy the same property. For more about the subject see Olevskii and Ulanovskii [2].

\section{Quasicrystals}

It is surprising that Meyer's concept of model sets turned out to be a recognized mathematical model for a physical phenomenon discovered much later. In the early 1980 s Dan Shechtman and his colleagues found aperiodic atomic structures whose diffraction patterns consist of isolated spots. For this discovery he received the 2011 Nobel Prize. Such a phenomenon has been perceived as a contradiction to classical crystallography. The structures got the name "quasicrystals."

A parallel with Meyer's model sets is clear: each $M$ is an aperiodic uniformly discrete set and it supports a measure $\mu$ with point spectrum. Since $\hat{f}$ in (1) is a Schwartz function, one can prove that the "visible" part of the spectrum, which is the set of spectral atoms whose size exceeds a fixed small number, is uniformly discrete (consists of spots!).

Similarly, discrete aperiodic measures with pure point spectrum are often called "Fourier quasicrystals." Meyer sets provide important examples of such sets.

On the other hand, if both the support and the spectrum of a measure $\mu$ in $\mathbb{R}^{n}$ are uniformly discrete, then it is a finite sum of translates and modulates of a Dirac comb, as proved by N. Lev and A. Olevskii [4]. Their result shows 


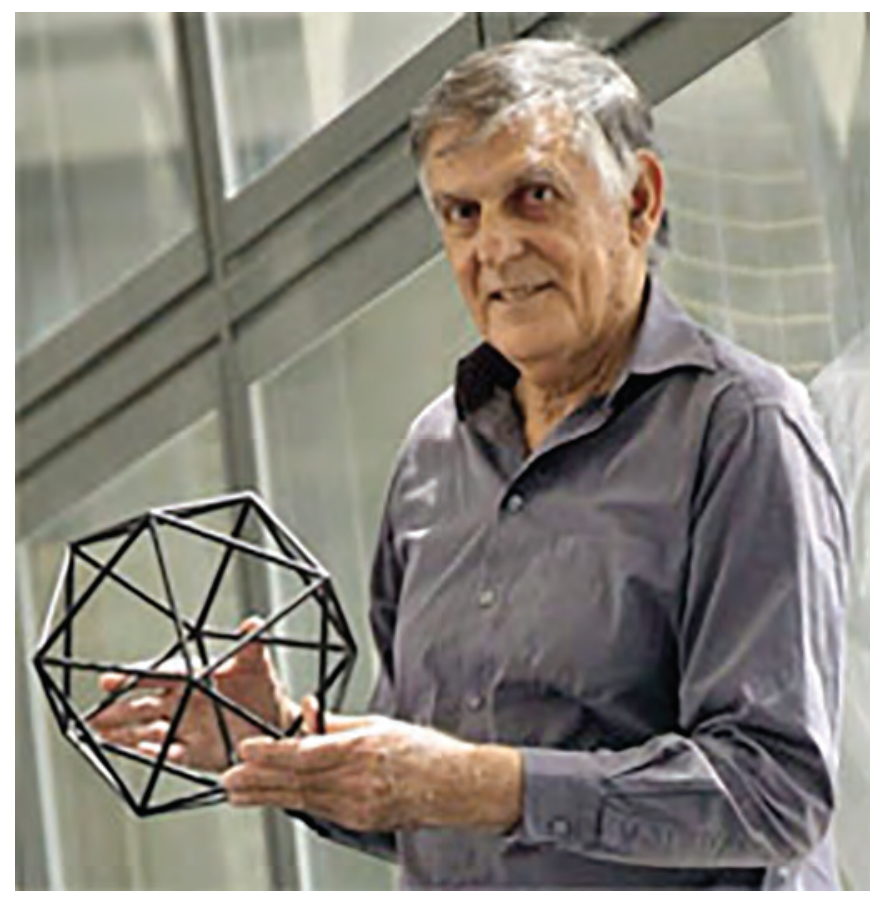

Dan Shechtman, Nobel Laureate for the discovery of quasicrystals, for which Meyer's model sets serve as mathematical models.

that in some natural situations a quasicrystal can not be observed.

For $n=1$ the result is true in full generality, in higher dimensions for positive measures. In particular, the support of such measures is periodic. This answered a problem raised by J. Lagarias [3].

\section{Nonclassical Poisson Formulas}

The characterization of measures as the sums of Dirac combs remains true even if the support of $\mu$ is just locally finite (whilst the spectrum is still uniformly discrete). On the other hand, there are measures such that both the support and the spectrum are locally finite but not periodic [4]. Further examples of this kind were found by M. Kolountzakis and Y. Meyer.

Each such measure generates a Poisson-type summation formula. A spectacular one, due to Meyer [5], has the form

$$
\hat{\mu}=c \mu \text {, }
$$

where $\mu$ is a purely atomic measure on $\mathbb{R}$ supported at the points $\pm|k+a|$, where $a \in \mathbb{R}^{3} \backslash \mathbb{Z}^{3}, k$ runs over $\mathbb{Z}^{3}$, and the masses of atoms are effectively defined.

Recently Meyer has moved forward substantially this line of research. He found a series of non-classic Poisson formulas with special arithmetics of the nodes.

Note that all examples known so far, in one way or another, are based on the classic Poisson formula. It would be interesting to know whether there are examples of a different nature.

\section{Cited Papers of Meyer}

[1] Y. MEYER, Algebraic numbers and harmonic analysis, NorthHolland, Amsterdam, 1972. MR0485769

[2] A. OleVSKII and A. UlANOVSKII, Functions with Disconnected Spectrum: Sampling, Interpolation, Translates, University Lecture Series, AMS, 2016. MR3468930

[3] J. C. LAGARIAS, Mathematical quasicrystals and the problem of diffraction. Directions in mathematical quasicrystals, In: CRM Monograph Series, vol. 13, pp. 61-93, AMS, 2000. MR1798989

[4] N. LEV and A. OLEvskiI, Quasicrystals and Poisson's summation formula, Invent. Math. 200 (2015), no. 2, 585-606. MR3338010

[5] Y. MEYER, Measures with locally finite support and spectrum, Proc. Natl. Acad. Sci. USA 113 (12) (2016) 3152-3158. MR3482845

\section{Photo Credits}

Figure 1 courtesy of YIT Corporation.

Photo of Dan Shechtman by Technion-Israel Institute of Technology (File:Shechtman_(2).jpg) [CC BY-SA 3.0 (https: //creativecommons.org/licenses/by-sa/3.0)], via Wikimedia Commons.

Photo of Alexander Olevskii used with his permission.

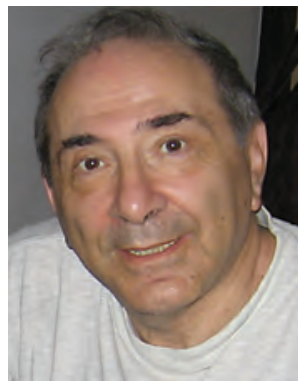

\section{Alexander}

Olevskii

\section{ABOUT THE AUTHOR}

Alexander Olevskii received his ScD degree in 1966 at Moscow State University. His research has been concentrated on problems of classical harmonic analysis and related areas.

\section{Albert Cohen}

\section{The Emergence of Sparse Analysis through the} Works of Yves Meyer

Since the turn of the century, the concept of sparsity has become prominent in many areas of applied mathematics, at the crossroad with other disciplines such as signal and image processing. Loosely speaking, sparse approximation refers to the possibility of representing certain complex objects by very few numbers up to a controlled loss of accuracy. This task is usually made possible once a proper mathematical representation of these objects has been provided, for example through a change of basis.

Such economical representations play an obvious role in data compression. They also found powerful applications in statistical estimation and inverse problems. The spectacular development of compressed sensing led to a deeper mathematical understanding of how sparsity

Albert Cohen is professor of mathematics at Laboratoire JacquesLouis Lions, Sorbonne Université, Paris, France. His email address is cohen@ann.jussieu.fr. 
could be exploited in many such applications. While Yves Meyer's work was not primarily focused on such areas, his contributions played a key role in the emergence and spreading of this concept and in the development of its mathematical foundations.

The present paper includes parts of a more detailed survey on the research works of Meyer published in the Abel volume [2], which can be consulted for further references.

\section{A Golden Decade}

The process of analyzing and representing an arbitrary function $f$ by means of more elementary functions has been at the heart of fundamental and applied advances in science and technology for several centuries. In more recent decades, implementation of this process on computers by fast algorithms has become of ubiquitous use in scientific computing. In the foundational example of the univariate Fourier expansions these elementary building blocks are the 1-periodic complex exponential functions defined by

$$
e_{n}(t)=e^{i 2 \pi n t}, \quad n \in \mathbb{Z},
$$

and they form an orthonormal basis of $L^{2}$ (]0,1[).

The functions $e_{n}$ are perfectly localized in frequency but have no localization in time, since their modulus is equal to 1 independently of $t$. This property constitutes a major defect when trying to efficiently detect the local frequency content of functions by means of Fourier analysis. It also makes Fourier representations numerically ineffective for functions that are not smooth everywhere. For example, the Fourier coefficients $c_{n}(f)$ of a 1-periodic piecewise smooth function $f$ with a jump discontinuity at a single point $t_{0} \in[0,1]$ decay like $|n|^{-1}$, which affects the convergence of the Fourier series on the whole of $\mathbb{R}$.

Wavelet bases are orthonormal bases of $L^{2}(\mathbb{R})$ with the general form

$$
\psi_{j, k}(x)=2^{j / 2} \psi\left(2^{j} x-k\right), \quad j \in \mathbb{Z}, k \in \mathbb{Z},
$$

where $\psi$ is a function such that $\int_{\mathbb{R}} \psi=0$. Due to their increased resolution as the scale level $j$ tends to $+\infty$, they are better adapted than Fourier bases for capturing local phenomena. The most basic example of the Haar system, which corresponds to $\psi=\mathrm{X}_{[0,1 / 2[}-\mathrm{X}_{[1 / 2,1[}$, has been known since 1911. In this example, the function $\psi$ suffers from a lack of smoothness, also reflected by the slow decay at infinity of its Fourier transform.

The construction of modern wavelet theory took place during the decade of 1980-1990. It benefited greatly from ideas coming from various (and sometimes completely disjoint) sources: theoretical harmonic analysis, approximation theory, computer vision and image analysis, computer aided geometric design, digital signal processing. One of the fundamental contributions of Yves Meyer was to recognize and organize these separate developments into a unified and elegant theory.

After some attempts to disprove their existence, Meyer turned the table in 1985 and gave a beautiful construction of orthonormal wavelet bases that belong to the Schwartz class

(3)

$$
S(\mathbb{R}):=\left\{f \in C^{\infty}(\mathbb{R}): \sup _{x \in \mathbb{R}}|x|^{k}\left|f^{(l)}(x)\right|<\infty, k, l \geq 0\right\},
$$

and are therefore well localized both in time and frequency. Another orthonormal wavelet basis with smoothness and localization properties had been obtained earlier in the work of Jan-Olov Strömberg. By its elegant simplicity, Meyer's construction was celebrated as a milestone.

A major turning point occurred in 1986 when Stéphane Mallat [5] introduced the natural framework that was the key to the general construction of wavelets, as well as to fast decomposition and reconstruction algorithms. A multiresolution approximation is a dense nested sequence of approximation spaces

$$
\{0\} \cdots \subset V_{j-1} \subset V_{j} \subset V_{j+1} \subset \cdots L^{2}(\mathbb{R}),
$$

generated by a so-called scaling function $\varphi$ in the sense that $\left(2^{j / 2} \varphi\left(2^{j}-k\right)\right)_{k \in \mathbb{Z}}$ is a Riesz basis of $V_{j}$. A countable family $\left(e_{k}\right)_{k \in \mathcal{F}}$ in a Hilbert space $V$ is called a Riesz basis if it is complete and there exists constants $0<c \leq C<\infty$ such that

$$
c \sum_{k \in \mathcal{F}}\left|x_{k}\right|^{2} \leq\left\|\sum_{k \in \mathcal{F}} c_{k} e_{k}\right\|_{V}^{2} \leq C \sum_{k \in \mathcal{F}}\left|x_{k}\right|^{2}
$$

holds for any finitely supported coefficient sequence $\left(x_{k}\right)_{k \in \mathcal{F}}$, and therefore by density for any sequence in $\ell^{2}(\mathcal{F})$.

In this framework, the generating wavelet $\psi$ is then constructed so that the functions $\left(2^{j / 2} \psi\left(2^{j}-k\right)\right)_{k \in \mathbb{Z}}$ constitute a Riesz basis for a complement $W_{j}$ of $V_{j}$ into $V_{j+1}$. This approach allowed in particular the construction of compactly supported orthonormal wavelets by Ingrid Daubechies [4].

The multiresolution analysis framework was immediately extended by Stéphane Mallat and Yves Meyer to multivariate functions, by tensorizing the spaces $V_{j}$ in the different variables. This leads to multivariate wavelet bases of the form

$$
\psi_{\varepsilon, j, k}^{\varepsilon}=2^{d j / 2} \psi_{\varepsilon}\left(2^{j} \cdot-k\right), \quad j \in \mathbb{Z}, k \in \mathbb{Z}^{d},
$$

for $\varepsilon=\left(\varepsilon_{1}, \ldots, \varepsilon_{d}\right) \in\{0,1\}^{d} \backslash\{(0, \ldots, 0)\}$, where

(7)

$\psi_{\varepsilon}\left(x_{1}, \ldots, x_{d}\right):=\psi_{\varepsilon_{1}}\left(x_{1}\right) \cdots \psi_{\varepsilon_{d}}\left(x_{x}\right), \quad \psi_{0}:=\varphi, \psi_{1}:=\psi$.

Adaptation of these bases to more general bounded domains of $\mathbb{R}^{d}$ as well as to various types of manifolds came in the following years, again based on the multiresolution concept.

All these developments are well documented in the classical textbooks [4],[8]. A major stimulus was the vision of powerful applications in areas as diverse as signal and image processing, statistics, and fast numerical simulation. This perspective was confirmed in the following decades. Meyer played a key role in identifying the mathematical properties that are of critical use in such applications, in particular the ability of wavelets to characterize a large variety of function spaces. As we next discuss, these properties naturally led to the concept of sparse approximation. He was also one of the first to point 
out some intrinsic limitations of wavelets and promote alternative strategies.

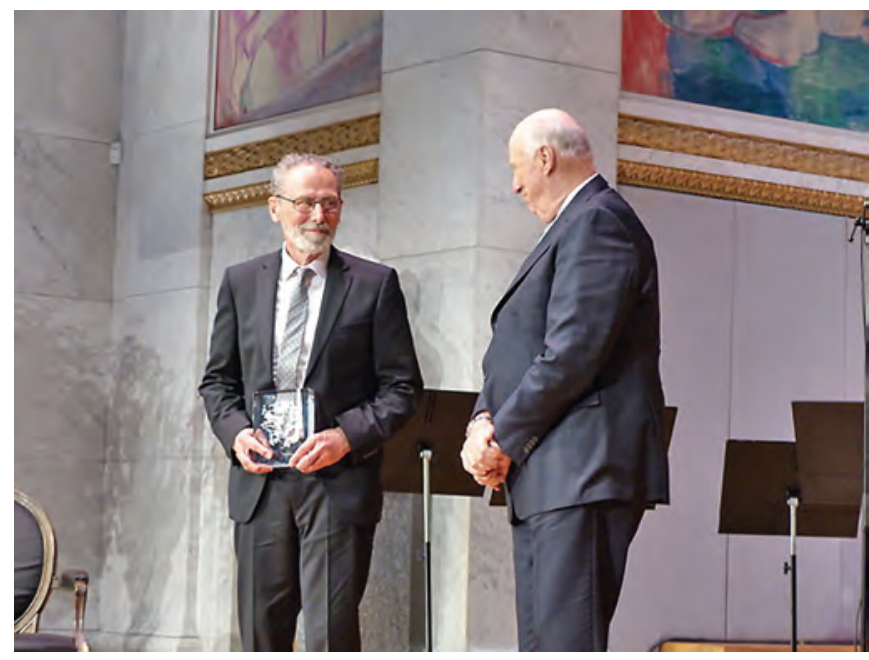

Yves Meyer receives Abel Prize from King Harald of Norway.

\section{Function Spaces and Unconditional Bases}

When expanding a function $f$ into a given basis $\left(e_{n}\right)_{n \geq 0}$, a desirable feature is that the resulting decomposition $f=\sum_{n \geq 0} x_{n} e_{n}$ is numerically stable: operations such as perturbations, thresholding or truncation of the coefficients $x_{n}$ should effect the norm of $f$ in a well-controlled manner. Such prescriptions can be encapsulated in the following classical property.

A sequence $\left(e_{n}\right)_{n \geq 0}$ in a separable Banach space $X$ is an unconditional basis if the following properties hold:

(i) It is a Schauder basis: every $f \in X$ admits a unique expansion $\sum_{n \geq 0} X_{n} e_{n}$ that converges towards $f$ in $X$.

(ii) There exists a finite constant $C \geq 1$ such that for any finite set $F \subset \mathbb{N}$,

(8)

$$
\left|x_{n}\right| \leq\left|y_{n}\right|, \quad n \in F \Longrightarrow\left\|\sum_{n \in F} x_{n} e_{n}\right\|_{X} \leq C\left\|\sum_{n \in F} y_{n} e_{n}\right\|_{X} .
$$

The property (8) means that membership of $f$ in $X$ only depends on the moduli of its coordinates $\left|x_{n}\right|$. In other words, multiplier operators of the form

$$
T: \sum_{n \geq 0} x_{n} e_{n} \rightarrow \sum_{n \geq 0} c_{n} x_{n} e_{n}
$$

should act boundedly in $X$ if $\left(c_{n}\right)_{n \geq 0}$ is a bounded sequence. Orthonormal and Riesz bases are obvious examples of unconditional bases in Hilbert spaces.

While the trigonometric system (1) is a Schauder basis in $L^{p}(] 0,1[)$ when $1<p<\infty$, it does not constitute an unconditional basis when $p \neq 2$, and it is thus not possible to characterize the space $L^{p}$ through a property of the moduli of the Fourier coefficients. The same situation is met for classical smoothness spaces, such as the Sobolev spaces $W_{\mathrm{per}}^{m, p}(] 0,1[)$ that consist of 1-periodic functions having distributional derivatives up to order $m$ in $L_{\text {loc }}^{p}$ : apart from the Hilbertian case $p=2$, for which one has

$$
f \in W_{\text {per }}^{m, 2}(] 0,1[) \Longleftrightarrow \sum_{n \in \mathbb{Z}}\left(1+|n|^{2 m}\right)\left|c_{n}(f)\right|^{2}<\infty,
$$

no such characterization is available when $p \neq 2$.

Meyer showed that, in contrast to the trigonometric system, wavelet bases are unconditional bases for most classical function spaces that are known to possess one. The case of $L^{p}$ spaces for $1<p<\infty$ is treated by the following observation: if the general wavelet $\psi$ has $C^{1}$ smoothness, the multiplier operator (9) by a bounded sequence belongs to a classical class of integral operators introduced by Calderón and Zygmund, which are proved to act boundedly in $L^{p}\left(\mathbb{R}^{d}\right)$. Conversely, Meyer showed that Calderón-Zygmund operators are "almost diagonalized" by wavelet bases in the sense that the resulting matrices have fast off-diagonal decay. This property plays a key role in the numerical treatment of partial differential and integral equations by wavelet methods.

The characterization of more general function spaces by the size properties of wavelet coefficients is particularly simple for an important class of smoothness spaces introduced by Oleg Besov. There exist several equivalent definitions of Besov spaces. The original one uses the $m$-th order $L^{p}$-modulus of smoothness

$$
\omega_{m}(f, t)_{p}:=\sup _{|h| \leq t}\left\|\Delta_{h}^{m} f\right\|_{L^{p}},
$$

where $\Delta_{h}^{m}$ is the $m$-th power of the finite difference operator $\Delta_{h}: f \mapsto f(\cdot+h)-f$. For $s>0$, any integer $m>s$, and $0<p, q<\infty$, a function $f \in L^{p}\left(\mathbb{R}^{d}\right)$ belongs to the space $B_{q}^{s, p}\left(\mathbb{R}^{d}\right)$ if and only if the function $g: t \rightarrow$ $t^{-s} \omega_{m}(f, t)_{p}$ belongs to $L^{q}\left(\left[0, \infty\left[, \frac{d t}{t}\right)\right.\right.$. One may use

$$
\|f\|_{B_{q}^{s, p}}:=\|f\|_{L^{p}}+|f|_{B_{q}^{s, p}} \text {, with } \quad|f|_{B_{q}^{s, p}}:=\|g\|_{L^{q}\left(\left[0, \infty\left[, \frac{d t}{T}\right)\right.\right.},
$$

as a norm for such spaces, also sometimes denoted by $B_{q}^{s}\left(L^{p}\left(\mathbb{R}^{d}\right)\right)$. Roughly speaking, functions in $B_{q}^{s, p}\left(\mathbb{R}^{d}\right)$ have up to $s$ (integer or not) derivatives $L^{p}$. The third index $q$ may be viewed as a fine tuning parameter, which appears naturally when viewing Besov spaces as real interpolation spaces between Sobolev space: for example, with $0<s<m$,

$$
B_{q}^{s}\left(L^{p}\right)=\left[L^{p}, W^{m, p}\right]_{\theta, q}, \quad s=\theta m .
$$

Particular instances are the Hölder spaces $B_{\infty}^{s, \infty}=C^{s}$ and Sobolev spaces $B_{p}^{s, p}=W^{s, p}$, when $s$ is not an integer or when $p=2$ for all values of $s$.

Let $\left(\psi_{\lambda}\right)$ denote a multivariate wavelet basis of the type (6), where for simplicity $\lambda$ denotes the three indices $(e, j, k)$ in (6). Denoting by $|\lambda|:=j=j(\lambda)$ the scale level of $\lambda=(e, j, k)$, we consider the expansion

$$
f=\sum_{|\lambda| \geq 0} d_{\lambda} \psi_{\lambda},
$$

where the coarsest scale level $|\lambda|=0$ also includes the translated scaling functions that span $V_{0}$.

The characterization of $B_{q}^{s, p}\left(\mathbb{R}^{d}\right)$ established by Meyer for such expansions requires some minimal prescriptions: one assumes that for an integer $r>s$ the univariate 
generating wavelet $\psi$ and scaling functions $\varphi$ that defines (6) have derivatives up to order $r$ that decay sufficiently fast at infinity, for instance faster than any polynomial rate, and that $\int_{-\infty}^{+\infty} t^{k} \psi(t) d t=0$ for all $k=0,1, \ldots, r-1$. Then, one has the norm equivalence

$$
\|f\|_{B_{q}^{s, p}} \sim\|\varepsilon\|_{\ell q}
$$

where the sequence $\varepsilon=\left(\varepsilon_{j}\right)_{j \geq 0}$ is defined by

$$
\varepsilon_{j}:=2^{s j} 2^{\left(\frac{d}{2}-\frac{d}{p}\right) j}\left\|\left(d_{\lambda}\right)_{|\lambda|=j}\right\|_{\ell^{p}} .
$$

A closely related characterization of Besov spaces uses the Littlewood-Paley decomposition

$$
f=S_{0} f+\sum_{j \geq 0} \Delta_{j} f, \quad \Delta_{j} f:=S_{j+1} f-S_{j} f,
$$

where $\widehat{S_{j} f}(\omega):=\Theta\left(2^{-j} \omega\right) \hat{f}(\omega)$ with $\Theta$ a smooth compactly support function with value 1 for $|\omega| \leq 1$. It has the same form as above, with now $\varepsilon_{j}:=\left\|\Delta_{j} f\right\|_{L^{p}}$. In the wavelet characterization the dyadic blocks are further discretized into the local components $d_{\lambda} \psi_{\lambda}$. Similar results have been obtained for Besov spaces defined on general bounded Lipschitz domains $\Omega \subset \mathbb{R}^{d}$ with wavelet bases adapted to such domains.

The norm equivalence (15) shows that membership of $f$ in Besov spaces is characterized by simple weighted summability properties of its wavelet coefficients. In the particular case $q=p$, this equivalence takes the very simple form

$$
\|f\|_{B_{p}^{s, p}} \sim\left\|\left(2^{\left(s+\frac{d}{2}-\frac{d}{p}\right)|\lambda|} d_{\lambda}\right)\right\|_{\ell^{p}} .
$$

As an immediate consequence, classical results such as the critical Sobolev embedding $B_{p}^{s, p} \subset L^{2}$ for $s=\frac{d}{p}-\frac{d}{2}$ take the trivial form of the embedding $\ell^{p} \subset \ell^{2}$ for $p<2$. While this embedding is not compact, an interesting approximation property holds: when retaining only the $n$ largest coefficients in the wavelet decomposition of $f$, the resulting approximation $f_{n}$ satisfies

$$
\left\|f-f_{n}\right\|_{L^{2}} \leq C n^{-r}\|f\|_{B_{p}^{s, p}}, \quad r:=\frac{s}{d}
$$

This follows immediately from the fact that, for $p<2$, the decreasing rearrangement of $\left(d_{k}\right)_{k \geq 1}$ of a sequence $\left(d_{\lambda}\right) \in \ell^{p}$ satisfies the tail bound

$$
\left(\sum_{k \geq n} d_{k}^{2}\right)^{1 / 2} \leq n^{\frac{1}{2}-\frac{1}{p}}\left\|\left(d_{\lambda}\right)\right\|_{\ell^{p}} .
$$

This last estimate shows that $\ell^{p}$ summability governs the compressibility of a sequence, in the sense of how fast it can be approximated by $n$-sparse sequences. The theory of best $n$-term wavelet approximation, generalizing the above remarks, has been developed by Ronald DeVore and his collaborators, in close relation with other nonlinear approximation procedures such as free knot splines or rational approximation.

A particularly useful feature of nonlinear wavelet approximation is that piecewise smooth signals, such as images, can be efficiently captured since the large coefficients are only those of the few wavelets whose supports contain the singularities. This is an instance of sparse approximation which aims to accurately capture functions by a small number of well chosen coefficients in a basis

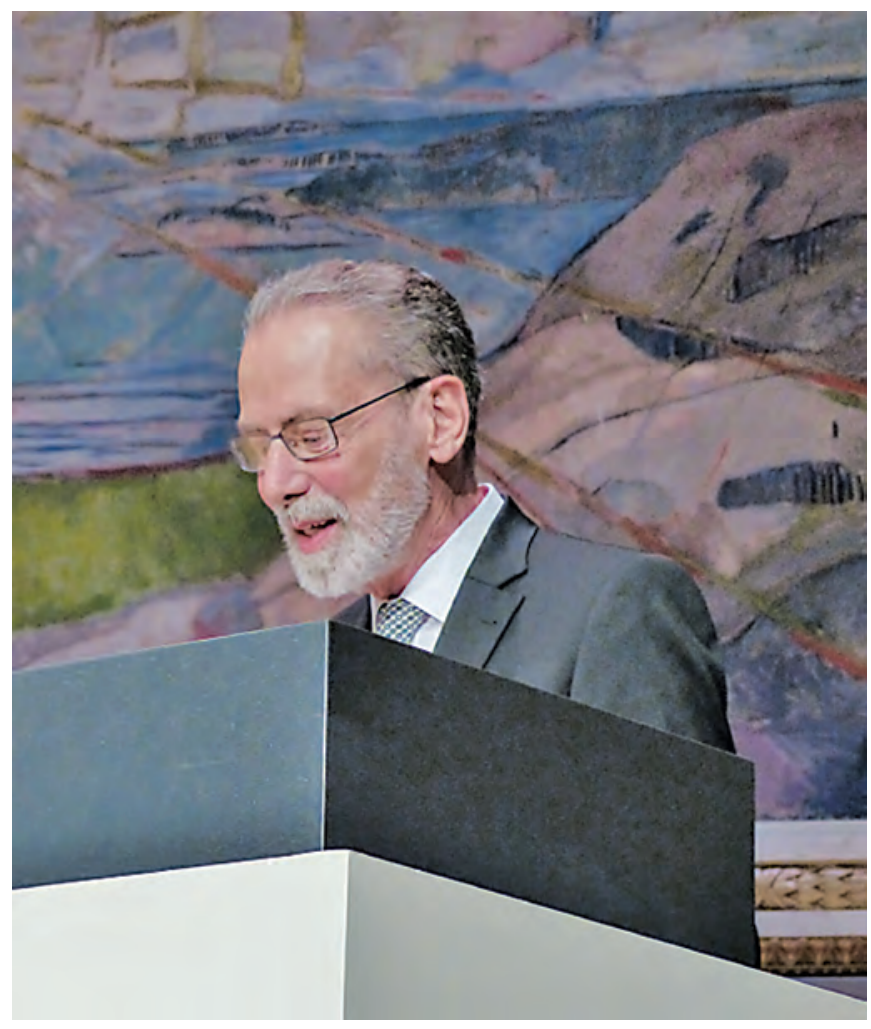

Yves Meyer at his Abel Prize Ceremony.

or dictionary expansion. Sparse approximation in unconditional bases was identified by David Donoho as a key ingredient for powerful applications in data compression and statistical estimation, in particular through thresholding algorithms that he developed jointly with Iain Johnstone, Gérard Kerkyacharian, and Dominique Picard. Pushed into the forefront by the work of Meyer, Donoho, and DeVore, sparse approximation became within a few years a prominent concept in signal processing and scientific computing.

\section{Taking Off from the Wavelet World}

The estimate (20) shows that the rate $n^{-r}$ of best $n$-term approximation of a function, using an orthonormal or Riesz basis $\left(\psi_{\lambda}\right)$, is implied by the $\ell^{p}$ summability of is coefficient sequence $\left(d_{\lambda}\right)$ with $\frac{1}{p}=r+\frac{1}{2}$. A more refined analysis shows that this rate is exactly equivalent to the slightly weaker property that $\left(d_{\lambda}\right)$ belongs to $w \ell^{p}$, which means that its decreasing rearrangement has the decay property

$$
d_{k} \leq C k^{-1 / p} .
$$

The spaces $\ell^{p}$ and $w \ell^{p}$ are thus natural ways of quantifying sparsity of a function when decomposed in an arbitrary orthonormal or Riesz basis $\left(\psi_{\lambda}\right)$ of a Hilbert space.

In the case of wavelet bases, these summability properties are equivalent to Besov smoothness. From an applicative point of view, a more natural question is: 
given a class of functions $\mathcal{K}$ in a Hilbert space, which basis should be used in order to obtain the sparsest possible representations of the element of this class? In view of the previous observations, this basis should be picked so that the coefficient sequence of any element of $\mathcal{K}$ belongs to $w \ell^{p}$, for the smallest possible value of $p$.

One class of particular interest for modeling real images is the space $\mathrm{BV}(Q)$ of bounded variation functions on the unit cube $Q:=[0,1]^{2}$ that consists of functions $f \in L^{1}(Q)$ such that $\nabla f$ is a finite measure. In particular, if $\Omega \subset Q$ is a set of finite perimeter, the characteristic function $\mathrm{X}_{\Omega}$ belongs to $\mathrm{BV}(Q)$. More generally, piecewise smooth images with edge discontinuities across curves of finite length have bounded variation. While the space $\mathrm{BV}(Q)$ admits no unconditional basis, we showed together with DeVore, Pencho Petrushev, and Hong Xu that it can be "almost" characterized by its decomposition in a bivariate wavelet basis $\left(\psi_{\lambda}\right)$ in the following sense: if $f=\sum d_{\lambda} \psi_{\lambda}$, one has

$$
\left(d_{\lambda}\right) \in \ell^{1} \Longrightarrow f \in \mathrm{BV}(Q) \Longrightarrow\left(d_{\lambda}\right) \in w \ell^{1} .
$$

In view of the previous remarks this shows that for general images of bounded variation, the rate of best $n$-term approximation in wavelet bases is $n^{-1 / 2}$. It can be shown that this rate is also the best that can be achieved by any basis. In particular, no polynomial rate can be achieved when using the Fourier basis. In this sense wavelets appear as the optimal tool for piecewise smooth images with edges of finite length.

The situation becomes quite different if one considers images with edges enjoying some geometric smoothness in addition to finite length. The simplest model consists of piecewise constant images with straight edges. For such images, Meyer [9] noticed that the decreasingly rearranged Fourier coefficients decay at rate

$$
c_{k} \leq C k^{-1} \log (k),
$$

therefore comparable to wavelet coefficients up to the logarithmic factor. When going to a higher dimensional cube $Q=[0,1]^{d}$, this rate persists for Fourier coefficients while wavelet representations become less effective.

A more elaborate model consists of the functions which are piecewise $C^{m}$ with edge discontinuities having $C^{n}$ geometric smoothness. For such classes $\mathcal{K}(n, m)$, both wavelet and Fourier decompositions can be outperformed by more sophisticated representations into functions that combine local support with directional selectivity. One representative example are the curvelets, introduced by Emmanuel Candès and Donoho, which have the form

(24) $\psi_{\lambda}=2^{3 j / 2} \psi\left(D^{j} R_{j}^{l} \cdot-k\right), \quad k \in \mathbb{Z}, \quad l=0, \ldots, 2^{j}-1$,

where $D$ is the anisotropic dilation matrix $\left(\begin{array}{ll}4 & 0 \\ 0 & 2\end{array}\right)$ and $R_{j}$ the rotation of angle $2^{-j-1} \pi$. The anisotropic scaling and angular selectivity allow to better capture the geometry of edges, leading to improved sparsity: for example, it is known that

$$
f \in \mathcal{K}(2,2) \Longrightarrow\left(d_{\lambda}\right) \in \ell^{p}, \quad p>2 / 3,
$$

where $d_{\lambda}$ are the coefficients of $f$ in the curvelet expansion. The value $2 / 3$ is optimal for this class. Other representation methods have since then been proposed and studied for better capturing geometry: contourlets, shearlets, bandlets, anisotropic finite elements.

Returning to univariate signals, one object of longterm interest to Meyer is signals whose "instantaneous frequency" evolves with time in some controlled manner. Such signals are called chirps and take the general form

$$
f(t)=\operatorname{Re}\left(a(t) e^{i \varphi(t)}\right),
$$

where $\left|\frac{a^{\prime}(t)}{a(t)}\right|<<\left|\varphi^{\prime}(t)\right|$ and $\left|\varphi^{\prime \prime}(t)\right|<<\left|\varphi^{\prime}(t)\right|^{2}$.

Typical examples of chirp are ultrasounds emitted by bats and recordings of voice signals, but the most famous one is the gravitational wave signal first detected in 2015, which has for a large part the behaviour

$$
f(t) \sim\left|t-t_{0}\right|^{-1 / 4} \cos \left(\left|t-t_{0}\right|^{5 / 8}+\varphi_{0}\right) .
$$

Wavelets are not the right tool for sparse representation of chirps. Time-frequency analysis such as the shorttime Fourier transform provides more natural tools, once proper orthonormal bases have been provided.

The first example of such a basis was originally suggested by Kenneth Wilson and formalized by Ingrid Daubechies, Stéphane Jaffard, and Jean-Lin Journé: an orthonormal basis of $L^{2}(\mathbb{R})$ is constructed by taking for all $n \in \mathbb{Z}$ the functions $\varphi_{0, n}(t)=\varphi(t-n)$ and

$$
\varphi_{l, n}(t)= \begin{cases}\sqrt{2} \varphi\left(t-\frac{n}{2}\right) \cos (2 \pi l t) & l \geq 0, l+n \in 2 \mathbb{Z}, \\ \sqrt{2} \varphi\left(t-\frac{n}{2}\right) \sin (2 \pi l t) & l>0, l+n \in 2 \mathbb{Z}+1 .\end{cases}
$$

The generating function $\varphi$ should satisfy certain symmetry properties. One possible choice is the scaling function associated with the orthonormal wavelet basis of Meyer, which is defined by $\hat{\varphi}=\sqrt{\kappa}$, where $\kappa$ is the symmetric and smooth cut-off function. A variant of this system, where the family is made redundant by additional dilations, was proposed in the papers of Sergei Klimenko and his collaborators for the sparse representation of gravitational waves and used for their detection.

In recent years, sparse approximation has also been intensively exploited for the treatment of high-dimensional approximation. Problems that involve functions of a very large number of variables are challenged by the so-called "curse of dimensionality": the complexity of standard discretization methods blows up exponentially as the number of variables grows. Such problems arise naturally in learning theory, partial differential equations, and numerical models depending on parametric or stochastic variables. Wavelet representations are not well suited for extracting sparsity in such high-dimensional applications. This motivated the development of better adapted tools, such as sparse grids, sparse polynomials, and sparse tensor formats. 


\section{Compressed Sensing and Quasi-Crystals}

The most usual approach for obtaining a sparse approximation of a discrete signal represented by a vector $x \in \mathbb{R}^{N}$ is to choose an appropriate basis, compute the coefficients of $x$ in this basis, and then retain only the $n$ largest of these, with $n<<N$.

This approximation process is adaptive since the indices of the retained coefficients vary from one signal to another. The view expressed by Candès, Justin Romberg, and Terence Tao [1] and Donoho is that since only a few of these coefficients are needed in the end, it should be possible to compute only a few non-adaptive linear measurements in the first place and still retain the information needed in order to build a compressed representation. These ideas have led since the turn of the century to the very active area compressed sensing.

If $m$ is the number of linear measurements, the observed data has the form

$$
y=\Phi x
$$

where $\Phi$ is an $m \times N$ matrix. Any $n$-sparse vector $x$ is uniquely characterized by its measurement if and only if no $2 n$-sparse vector lies in the kernel of $\Phi$. In other words, any submatrix of $\Phi_{T}$ obtained by retaining a set $T \subset\{1, \ldots, N\}$ of columns with $\#(T)=2 n$ should be injective. It is easily seen that a generic $m \times N$ matrix satisfies this property provided that $m \geq 2 n$, and therefore $m=2 n$ linear measurements are in principle sufficient to be able to reconstruct $n$-sparse vectors. However, the reconstruction from $2 n$ measurements will generally be computationally untractable when $N$ is large and unstable due to the fact that $\Phi_{T}^{*} \Phi_{T}$, even if invertible, can be very ill-conditioned.

Stability and computational feasibility can be recovered at the expense of a stronger condition introduced by Candès, Romberg, and Tao: the matrix $\Phi$ satisfies the restricted isometry property (RIP) of order $k$ with parameter $0<\delta<1$ if and only if

$$
(1-\delta)\|z\|_{2}^{2} \leq\left\|\Phi_{T} z\right\|_{2}^{2} \leq(1+\delta)\|z\|_{2}^{2}, \quad z \in \mathbb{R}^{k},
$$

for all set $T \subset\{1, \ldots, N\}$ such that $\#(T)=k$. Under such a property with $k=2 n$ and $\delta<1 / 3$, it was shown that an $n$-sparse vector can be stably reconstructed from its linear measurements by a convex optimization algorithm, which consists in searching for the solution of (29) with minimal $\ell^{1}$-norm.

Measurement matrices $\Phi$ of size $m \times N$ that satisfy RIP to order $k$ are known to exist in the regime $m \sim k \log (N / k)$. Therefore, with $k=2 n$ the measurement budget $m$ is linear in $n$ up to logarithmic factors. However the constructions of such matrices rely upon probabilistic arguments: they are realizations of random matrices for which it is proved that RIP of order $2 n$ holds with high probability under this type of regime. Two notable examples are the matrix with entries consisting of independent centered Gaussian variables of variance $1 / m$ and the matrix obtained by picking at random $m$ rows from the $N \times N$ discrete Fourier transform matrix. The currently available deterministic constructions of matrices satisfying RIP of order $k$ require the non-optimal regime $m \sim k^{2}$ up to logarithmic factors.

In recent years, Meyer studied the problem of sampling continuous bandlimited signals with unknown Fourier support, which may be viewed as an analog counterpart to the above compressed sensing problem. For any set $E \subset \mathbb{R}^{d}$, we denote by $\mathcal{F}_{E}$ the Paley-Wiener space of functions $f \in L^{2}\left(\mathbb{R}^{d}\right)$ such that their Fourier transform

$$
\hat{f}(\omega)=\int_{\mathbb{R}^{d}} f(x) \exp (-i 2 \pi \omega \cdot x) d x,
$$

is supported on $E$. Sampling theory for such functions has been motivated since the 1960s by the development of discrete telecommunications. It is well known, since the foundational work of Claude Shannon and Harry Nyquist, that regular grids, which are full-rank lattices

$$
L=B \mathbb{Z}^{d},
$$

where $B$ is a $d \times d$ invertible matrix, are particularly suitable for the sampling of certain band-limited functions. This may be seen as a direct consequence of the Poisson summation formula, which says that for any sufficiently nice function $f$,

$$
|L| \sum_{\lambda \in L} f(\lambda) e^{i 2 \pi\langle\lambda, \omega}=\sum_{\lambda^{*} \in L^{*}} \hat{f}\left(\omega+\lambda^{*}\right) .
$$

Here $|L|:=|\operatorname{det}(B)|$ is the measure of the fundamental volume of $L$, and $L^{*}=\left(B^{t}\right)^{-1} \mathbb{Z}^{d}$ its dual lattice. This formula shows that, if $E \subset \mathbb{R}^{d}$ is a compact set with translates $\left(E+\lambda^{*}\right)_{\lambda^{*} \in L^{*}}$ having intersections of null measure, functions with Fourier transform supported in $E$ are then stably determined by their sampling on $L$. Such sets $E$ should in particular have measure smaller than the density of $L$, that is,

$$
|E| \leq \operatorname{dens}(L):=\left|L^{*}\right|=|L|^{-1} .
$$

One elementary example, for which equality holds in the above, is the fundamental volume of the lattice $L^{*}$, that is,

$$
E_{L^{*}}=\left(B^{*}\right)^{-1}\left([0,1]^{d}\right),
$$

or any of its translates.

A theory of stable sampling on more general discrete sets was developed in the 1960s by Henry Landau and Arne Beurling. The possibility of reconstructing any $f \in \mathcal{F}_{E}$ from its samples over a discrete set $\Lambda \subset \mathbb{R}^{d}$ is described by the property of stable sampling: there exists a constant $C$ such that

$$
\|f\|_{L^{2}}^{2} \leq C \sum_{\lambda \in \Lambda}|f(\lambda)|^{2}, \quad f \in \mathcal{F}_{E} .
$$

Landau proved that a necessary condition for such a property to hold is that

$$
\operatorname{dens}(\Lambda) \geq|E| \text {, }
$$

where

$$
\operatorname{dens}(\Lambda)=\liminf _{R \rightarrow \infty} \inf _{x \in \mathbb{R}^{d}} \frac{\#(\Lambda \cap B(x, R))}{|B(x, R)|}
$$

is the lower density of $\Lambda$, which is the usual density $\operatorname{dens}(\Lambda)$ when the standard limit exists. 


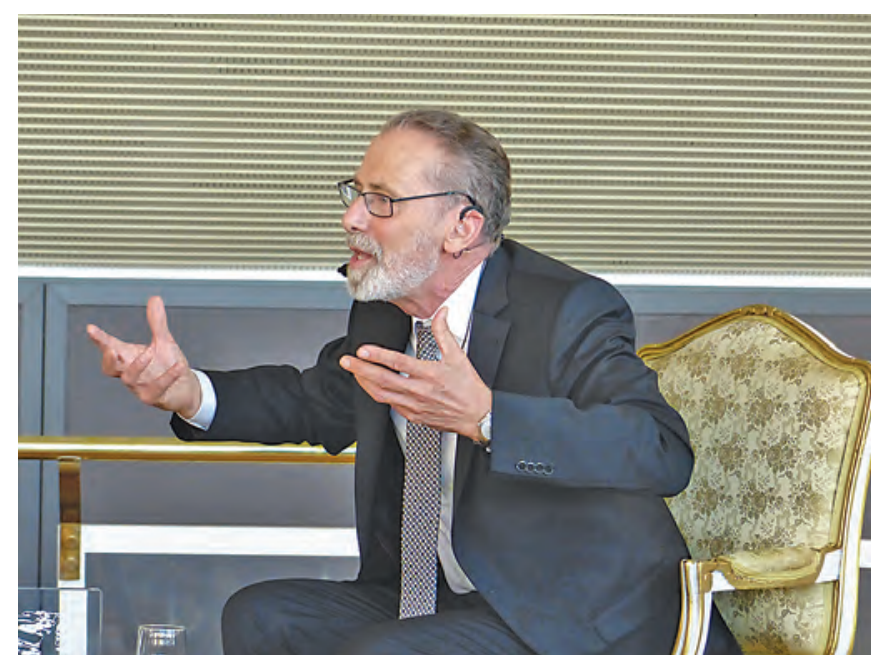

Yves Meyer giving an exuberant interview post Abel Prize Ceremony.

A continuous signal is $s$-sparse in the Fourier domain if it belongs to $\mathcal{F}_{E}$ for some set of Lebesgue measure $|E| \leq s$. Stable reconstruction of any $s$-sparse signal from its sampling on a discrete set $\Lambda$ requires that this set has the property of stable sampling for all sets of measure $|E| \leq r:=2 s$. Such sets $\Lambda$ are called universal sampling sets. Obviously, they should have density larger than $r$, but this condition cannot be sufficient. The case of a regular lattice $L$ is instructive: on the one hand, the set $E_{L^{*}}$ has measure $\left|E_{L^{*}}\right|=|L|^{-1}=\operatorname{dens}(L)$ and satisfies the stable sampling property in view of (33). On the other hand, other sets $E$ with the same or even smaller measure could have their translates by $\Lambda^{*}$ overlapping with nonzero measure, which is a principle obstruction to these properties. This phenomenon is well known in electrical engineering as aliasing. This shows that universal sampling sets cannot be regular lattices.

Alexander Olevskii and Alexander Ulanovskii gave the first construction of a set $\Lambda$ of uniform density that has the stable sampling property for any set $E$ such that

$$
|E|<\operatorname{dens}(\Lambda) \text {. }
$$

Meyer had the intuition that the mathematical models of quasicrystals that emerged from his early work on harmonic analysis and number theory [7] could provide a natural alternative solution to this problem.

One such model is obtained by the following cut and project scheme that was implicit in earlier work on algebraic number theory: the set of interest is obtained by projecting a "slice" cut from a higher-dimensional lattice in general position. More precisely, if $L$ is a full rank lattice of $\mathbb{R}^{d+m}$ for some $d, m>0$, we denote by $p_{1}(x) \in \mathbb{R}^{d}$ and $p_{2}(x) \in \mathbb{R}^{m}$ the components of $x \in \mathbb{R}^{d+m}$ such that $x=\left(p_{1}(x), p_{2}(x)\right)$ and assume that $p_{1}$ is a bijection between $L$ and $p_{1}(L)$ with dense image. A similar property is assumed for $p_{2}$. Let $K \subset \mathbb{R}^{m}$ be a Riemann integrable compact set of positive measure. The associated model

set $\Lambda=\Lambda(L, K) \in \mathbb{R}^{d}$ is defined by

$$
\Lambda:=\left\{p_{1}(x): x \in L, p_{2}(x) \in K\right\} .
$$

The density of a model set $\Lambda=\Lambda(L, K)$ is uniform and given by

$$
\operatorname{dens}(\Lambda)=\frac{|K|}{|L|} .
$$

Basarab Matei and Meyer [6] showed that the stable sampling property holds for any $E$ under the condition (39) for model sets $\Lambda:=\Lambda(L, K) \subset \mathbb{R}^{d}$ such that $K$ is a univariate interval. Such sets are called simple quasicrystals. They are universal sampling sets and may therefore be used for the reconstruction of $s$-sparse signals with $2 s<\operatorname{dens}(\Lambda)$. A remarkable fact is that, in contrast to compressed sensing matrices, their construction does not rely on any probabilistic argument.

\section{Cited Papers of Meyer}

[1] E. CANDÈs, J. Romberg and T. TAO, Stable signal recovery from incomplete and inaccurate measurements, Comm. Pure Appl. Math. 59, 1207-1223, 2005. MR2230846.

[2] A. CoHen, A journey through the mathematics of Yves Meyer, The Abel Volume, 2018.

[3] I. DAUBECHIES, Orthonormal bases of compactly supported wavelets, Comm. Pure and Appl. Math. 41, 909-996, 1988. MR951745

[4] I. DAUBECHIES, Ten lectures on wavelets, SIAM, Philadelphia, 1992. MR1162107

[5] S. MALLAT, Multiresolution approximation and wavelet orthonormal bases of $L^{2}(\mathbb{R})$, Trans. Amer. Math. Soc. 315, 69-88, 1989. MR1008470

[6] B. MATEI and Y. MEYER, Simple quasicrystals are sets of stable sampling, Journal of Complex Variables and Elliptic Equations 55, 947-964, 2010. MR2674875

[7] Y. MEYER, Algebraic numbers and harmonic analysis, NorthHolland, New York, 1972. MR0485769

[8] Y. MeYER, Wavelets and Operators, vol. 1, Cambridge University Press, 1997. MR1228209

[9] Y. MEYER, Oscillating patterns in image processing and in some nonlinear evolution equations, Jacqueline Lewis Memorial Lectures, AMS, 2001. MR1852741

\section{Photo Credits}

Photos of Yves Meyer courtesy of Stéphane Jaffard.

Author photo courtesy of Albert Cohen.

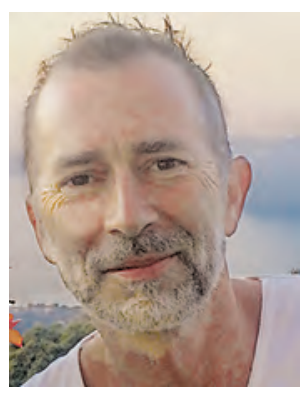

Albert Cohen

\section{ABOUT THE AUTHOR}

Albert Cohen's current research interests include nonlinear and high-dimensional approximation theory, statistics, signal-imagedata processing, numerical analysis, and inverse problems. 